AperTO - Archivio Istituzionale Open Access dell'Università di Torino

\title{
The ontology of drama
}

\section{This is the author's manuscript}

Original Citation:

Availability:

This version is available http://hdl.handle.net/2318/1690518

since 2019-02-06T10:43:09Z

Published version:

DOI:10.3233/AO-190204

Terms of use:

Open Access

Anyone can freely access the full text of works made available as "Open Access". Works made available under a Creative Commons license can be used according to the terms and conditions of said license. Use of all other works requires consent of the right holder (author or publisher) if not exempted from copyright protection by the applicable law. 


\title{
The ontology of drama
}

\author{
Rossana Damiano $^{\mathrm{a}, *}$, Vincenzo Lombardo ${ }^{\mathrm{a}}$ and Antonio Pizzo ${ }^{\mathrm{b}}$ \\ ${ }^{a}$ Dipartimento di Informatica and CIRMA, Università di Torino, Italy \\ E-mails: rossana.damiano@unito.it,vincenzo.lombardo@unito.it \\ ${ }^{\mathrm{b}}$ Dipartimento di Studi Umanistici and CIRMA, Università di Torino, Italy \\ E-mail:antonio.pizzo@unito.it
}

Abstract. In this paper, we describe the ontology of drama called Drammar. Pervasive in old and new media, and relevant from a multi-disciplinary perspective that ranges from literary criticism and semiotics to aesthetics and psychology, the manifestations of drama are ubiquitous in today's culture, overcoming the boundaries of genres and formats. Drawing from an extensive survey of the literature on drama, Drammar formalizes the elements of drama in a media- and task-independent way. Drammar is encoded in OWL2 to provide an interoperable formal model for representing and studying drama in a variety of systems. Issued by a research initiative carried out in an interdisciplinary way over a decade, Drammar abstracts from the media and languages by which drama is conveyed, providing a formal systematization of the core notions about drama that leverages the theories and models of agency in the tradition of Artificial Intelligence. In this paper, we illustrate the ontology, describing its design and motivations through examples and use cases.

Keywords: Drama ontology, formal representation of drama, media annotation

Accepted by: Roberta Ferrario

\section{Introduction}

In its wider meaning, drama encompasses different media and languages, ranging from Greek tragedy and musical drama to action movies and video games: despite their huge differences, these representational formats share traits of the cultural construct that we recognize as "drama", such as characters, actions, and conflicts. The exponential spread of drama in contemporary culture has led Esslin (1988) to forge the definition of "dramatic media", i.e. media that display characters performing actions; they include theatre, but also cinema and television. As a form of intangible cultural heritage, drama is characterized by an evolving nature, with form and function that change in time: consider, for example, the difference between the Greek Tragedy Oedipus and the modernist play Six Characters in Search of an Author.

While the concrete manifestations of drama are screenplays, theatrical performances, radio dramas, movies, etc., the dramatic content underlying these manifestations does not depend on the specific medium or language: take, for example, the Arden edition of the textual drama Hamlet ${ }^{1}$ and Laurence Olivier's movie Hamlet ${ }^{2}$ two examples of the dramatic media heritage that share a similar dramatic content despite the difference of the media support: Arden Hamlet is in iambic English, while the derivative work devised by Lawrence Olivier is an audiovisual expression that relies on filmic language.

\footnotetext{
${ }^{*}$ Corresponding author. E-mail: rossana.damiano@unito.it.

${ }^{1}$ Hamlet. The Arden Edition of the Works of William Shakespeare. 1982. Harold Jenkins (Editor), Thomson Learning.

${ }^{2}$ Hamlet, Two Cities production, 1948.
} 
This expressivity of drama in several media has established a need for representational tools that can encompass the dramatic contents in a way that is independent of the specific media.

In this paper, we describe an ontology of drama, called Drammar, aimed at representing the knowledge about drama in a machine-readable format, to serve the task of encoding dramatic contents coherently across different media and languages. In particular, Drammar addresses the so-called dramatic qualities, that is, those elements that are necessary for the existence of a drama, shared by a number of analyses in drama scholarship. Bazin speaks of "dramatic elements" as "interchangeable between one art and another" (Bazin and Gray, 1967). For drama, we intend "an action played live by characters" (Szondi, 1983): drama must show some characters in their actions and, differently from narrative, the focus is less on the descriptions of feelings, attitudes or actual movements, and the action must be rendered directly as the manifestation of some intention, as clearly stated by Styan (1963). In fact, actions, organized into bounded segments, stem from characters' internal motivations, providing information on the characters themselves and their goals. In order to achieve its representational goals, Drammar provides patterns for describing the main constituents of drama, namely characters (or agents), the action conveyed to the audience, and the conflicts emerging from their opposed intentions, orchestrated in a well formed, culturally acknowledged structure.

Drammar addresses several professional roles. The benefits for the theorist stem from the verification, through the encoding of drama in formal terms, of the features of the realizations of drama (such as text and authorship) put forth by drama studies; for the media designer, from the availability of automatic reasoning tools for appraising the dramatic qualities of media; for the scholar in AI, from the availability of a formal specification for devising processing and generation tools; for the community of drama scholars and professionals, from the availability of a theoretical model of drama, unambiguously described in formal terms, that does not leverage literary values (such as style, prose, verse).

Drammar is the outcome of an interdisciplinary research initiative carried out over a decade. Its consistency has been checked against the annotation of drama (i.e., the encoding in Drammar of the content of dramatic works) and the derived visualization, employed by scholars for purposes of didactics and research. Lombardo and Pizzo (2016) introduced a graphical interface for the annotation and visualization of drama that we applied to the analysis of Stanislavski's Action Analysis in Albert et al. (2016); Lombardo et al. (2015b) coupled an earlier release of the ontological representation with a rule-based calculation of characters' emotions, verified against manual annotation provided by students and scholars (Lombardo et al., 2015a); Lombardo et al. (2016) proposed the use of Drammar to safeguard drama as a form of intangible cultural heritage, underlying the tangible media (Lombardo et al., 2017b). In 2018, the Drammar ontology resource was released with a conference paper (Lombardo et al., 2018), with a permanent URL address and the registration to the Linked Open Vocaularies (LOV) platform. ${ }^{3}$ In this contribution, we address specifically the design of Drammar, accounting for the commitments that have informed its design and describing the development of the ontology from a set of elements distilled from drama scholarship.

The plan of the paper is the following: in Section 2, after illustrating the motivations for undertaking the compilation of an ontology of drama, we survey the sources of knowledge about drama that were the input to Drammar. The representational foundations of the ontology, external to drama, are described in Section 3. In Section 4, we illustrate the ontology engineering process. Section 5 describes the ontology; its use for drama representation is exemplified in Section 6. In Section 7, we briefly illustrate the use of Drammar for annotation as part of some practical projects that address the needs of drama scholars and

\footnotetext{
${ }^{3}$ https://lov.linkeddata.es
} 
practitioners, discussing the status of the annotations with respect to the original works. Related work (Section 8) and conclusion end the paper.

\section{Background and motivations}

In the last decade, the emerging technologies for media indexing and retrieval, mainly geared to multimedia contents, have addressed the markup of narrative texts, thus prompting a number of initiatives that leverage structured semantic representations. In particular, the pioneering Narrative Knowledge Representation Language (NKRL) project combines the use of markup for the encoding of the narrative content of text with the use of frames to represent the narrated story incidents (Zarri, 1997, 2014). As part of the more general trend of constructing resources for the automation of language processing, Elson introduced a template-based language for describing the content of narrative texts, with the goal of creating a corpus of annotated stories, called DramaBank (Elson, 2012). The annotation of narrative text has recently evolved towards minimal annotation schemas targeted at grasping the regularities of written and oral narratives at the discourse level, as exemplified by Rahimtoroghi et al. (2014). Narrative annotation projects, being tailored to the representation of stories in text, tend to focus on the expressive characteristics of this medium, so the schemata they put forth can be only partially extended to other media. In addition, they fall short of identifying the dramatic qualities of narratives, since they rely on a different tradition, i.e. narratology.

In parallel with the trend sketched above, narrative annotation has extended to non-textual media, thanks also to the languages and resources made available by the Semantic Web project (Berners-Lee et al., 2001). A media-independent model of story is provided by the OntoMedia ontology, exploited in the Contextus Project (Jewell et al., 2005a; Lawrence, 2011) to annotate the narrative content of media objects ranging from written literature to comics and TV fiction. This project encompasses some concepts that are relevant for the description of drama, such as, e.g., the notion of character; mainly targeted at the comparison of story events and timelines across media, it lacks the capability of representing the core notions of drama, such as the notion of conflict. The StorySpace ontology (Wolff et al., 2012) is an ontology of story aimed at supporting museum curators in linking the content of artworks through stories, with the ultimate goal of enabling the generation of user-tailored content retrieval (Mulholland and Collins, 2002). However, the representation of story provided by StorySpace is functional to curating activities; as such, it is not committed to a comprehensive account of the narratological theory; moreover, it lacks some crucial elements for drama ontology, such as the notion of character.

All the initiatives surveyed above, though relevant at the theoretical and methodological level, address the notion of story, so they lack the capability to represent the dramatic qualities of drama manifestations through media. Any drama, beyond the form it takes, produces in the audience the perception of something, intuitively called "story", directly enacted by its characters. The story is the set of all the events in a drama, both those presented explicitly and those inferred by the audience. The story of Hamlet includes both the dispute between two reigns (Denmark and Norway), and the murder of the King; nonetheless, Shakespeare has selected a finite set of events and actions in order to represent them through the enacted drama, and has left the other parts to the character's telling. Styan (1963), in his essay The Elements of Drama, discussed the difference between literature and drama: his contribution, among others, assigns a leading role to intentionality. According to Styan, drama takes actions "along a planned course, a 'line of intentions'. This is not a question of 'plot'." (Styan, 1963, p. 121). The goal of this well orchestrated, "planned course" is to let characters' conflicts emerge, as acknowledged since the 
18th century by Hegel, who identified the core of drama in the collisions of characters' passions. The same line was reiterated two centuries later by drama writing theorists, such as Egri (1946) and Field (2003). The former synthesizes his dramatic writing technique with the notion of "premise": stated from the characters and their conflicts, it provides a unitary direction to the unfolding of drama. The latter identifies the dramatic qualities of the plot in the characters' attempts to overcome the obstacles that get in their way.

There are multiple theories about drama, ranging from formal to speculative. A large part of the literature on drama intends drama as a written, or literary work, subject to an analysis that can proceed according to categories such as style, vocabulary, genre. This trend has run throughout the twentieth century to the present day, often blurring the boundaries between literary criticism and cultural studies (see, for example, the seminal work on Shakespeare' characters by Bloom (1998)). In opposition to this trend, which focuses on specific drama manifestations, the formal approach to drama investigates the nature of dramatic elements across all manifestations of drama. The formal approach to drama can be traced back to Aristotle's Poetics (2013) and his description of the six formative elements of the tragedy. Albeit dispersed and not yet unitary, this approach has produced a large body of works especially in the last two centuries. According to Esslin:

It thus seems legitimate to attempt an examination of the whole field of drama. That is: to try and describe the ways, common to all dramatic media, by which they achieve their dramatic effects, i.e. those effects that derive from the mimesis of human interaction through its embodiment by human beings assuming and presenting the identities of (fictional or real, but 'historical') human beings and presenting this interaction to an audience, as though it was happening at the very moment before their own eyes (Esslin, 1988, p. 33).

The peculiarity of this view on drama is well summarized in a mid-twentieth century textbook from Cleanth Brooks and Robert B. Heimlan, where the authors want to define the fundamental structure of drama as a special form with methods and characteristics of its own (Brooks and Heilmann, 1946). Even if we do not aim to survey the whole body of this kind of literature on drama here, it is still possible to enumerate a few aspects addressed by scholars: Szondi (1983) defines drama as an action at present time acted by characters; Lavandier (1994) stresses the recursiveness of drama structure; Egri (1946) indicates that the emotional tensions is the criterion that drives the organization of dramatic events in the plot; Ryngaert (2008) places the notion of conflict as the key element for the analytic apprehension of the dramatic text. The ontology Drammar encompasses a media independent notion of drama, aimed to identify the elements shared by different, cross-media manifestations of drama, which include Shakespeare and videogames within the same domain, avoiding references to style, artistic qualities, and even interpretation. Following a sort of "engineering" or "technical" approach, we focused on structural elements as the starting point for recognizing a shared model of dramatic writing: how characters are engaged, which conflicts take place, how the plot is articulated. Notice that this program does not go so far as identifying a limited number of functions, or roles, as postulated by the semiotic tradition represented, among others, by Propp (1968) and Greimas (1977). Following the suggestion expressed by Elam, our goal, here, is to formalize a theory of dramatic action (Elam, 1980), which may subsequently accommodate in its framework an actantial structure drawn from semiotic studies. A relevant contribution in this sense is provided by the narrative ontology proposed by Ciotti (2016): mainly based on the tradition of semiotic and structuralist narratology, it contains key notions such as Actant, Action, and Actor. 
The use of the computational ontology format not only allows the encoding of the conceptual model of drama in a formal, unambiguous way, as called for by Varela (2016), but also makes the knowledge about drama both available as a vocabulary for the interchange of annotations across different projects and readily usable as a representational tool for applications that process and manipulate these annotations in automatic ways. Benefits of annotation range from the possibility of detecting and measuring regularities in drama, useful for comparative studies, to the automatic generation of drama, which may leverage annotated corpora to combine dramatic elements into new objects according to the formal model of drama expressed in the ontology.

Bearing in mind that the cultural object called "drama" includes many features (such as genre, topic, writing style, and even Weltanschauung ${ }^{4}$ ), we focus on the elements that have been stated as necessary in the literature, namely action, agent, conflict, and segmentation.

The word "action" signifies an intentional, purposive, conscious and subjectively meaningful activity. It is done by an agent and it is the expression of a will, thus involving a goal, an intentionality. It is a key concept of dramatic theory: the etymological roots of the words "drama" and "dramaturgy" themselves stem from the ancient Greek verb drào, which means "to do", "to act", intended as performing an action following a human deliberation. Action is a dynamic element that determines the unfolding of situations in a drama, creating transitions among the phases of the drama; spanning onto multiple extents, it connects and gives coherence to all drama parts (Aristotle, 2013; Olson, 1961; Spencer, 2002; Szondi, 1983).

In our modelling, we employ the term "agent" rather than "character" for two reasons. First, we are not interested here in the psychological, moral, social or political entity that comes out of the drama as a cognitive product of the audience; our formal approach is concerned with the structural elements of the dramatic action and is not meant to address the interpretation of that action, intended as the action type and quality. Second, since we focus on the dramatic action as the core of drama, the notion of agent accounts for someone who primarily does the actions, so that all the other outcomes of its activity are mediated by its actions. From our modelling point of view, the notion of agent does not take into account the historical differences between the hero in Greek Tragedy, the romantic protagonist, or the modern character, but we focus on its qualities as agent. Currie (2009) describes the character as an entity that has intentions and mental states, so to motivate his/her behavior, a line also supported by contemporary models of scriptwriting such as those put forth by Egri (1946), McKee (1997), Seger (1990) and Lavandier (1994).

Conflict is a fundamental principle of dramatic theory. Indeed, the main characteristic of dramatic action is the expression of a tension, achieved through the opposition of characters, i.e. an attitude towards the creation of contrasts, or "conflicts". Contrasts become manifest in some situation of conflict that occurs in drama, which is characterized by an obstacle that obtrudes the achievement of the character's commitment. Conflict is traditionally indicated as the force that motivates the character's changes. The notion of conflict is ubiquitous in the history of dramatic theory and critics, from ancient tragedy on. Nevertheless, it reached its modern meaning only during the growth of the new "serious genre" (late 18th century), when it took on more specific and definite traits. From there on, it has permeated the modern drama.

Since its origins, dramatic theory has considered drama as a unitary whole, provided with proper length and made of different parts. Consequently, it should be possible to segment the dramatic works

\footnotetext{
${ }^{4}$ In the Dictionary of Literary Terms by Cuddon (2012), Weltanschauung is defined as "a particular author's attitude to the world, or to the prevailing spirit and vision of a period" (p. 770).
} 
into parts, and analyze how these are organized together in order to create the wholeness of the work. Recognizing these parts implies defining both the rules of segmentation, and the identification of the elements. The classical division of the tragedy into five parts dates back to Horace's Ars Poetica (Horace, 1989), where he theorized dramatic plays as a five-act construction, and lasted several centuries. Nevertheless, since the work of Freytag (1863), it is clear that a drama can be subdivided into parts that do not address practical reasons (such as characters' entrances or exits) but rather dramatic reasons, such as phases of the action. The literature has adopted different naming rules for the segmentation (beats, scene, sequence, acts, episode); following Lavandier (1994), who resorts to the term "dramatic node" to describe the basic element of this fractal structure, we adopt the generic term of unit. In our model of drama, units are the containers of the characters' actions, which result from their deliberations. Units follow each other according to a specific timeline, but their organization does not necessarily correspond to a sequential and linear unfolding in time. Since units are complex elements, they involve reciprocal relationships, both with other units located at the same structural level, or at other higher or lower structural levels.

\section{Conceptual foundations for drama representation}

Drammar is the result of a two-fold design effort:

- A top down systematization of the dramatic elements into the high-level categories provided by foundational ontologies (Masolo et al., 2002; Pease et al., 2002), which draw from the philosophical tradition in ontology. The reference to the high-level categories acknowledged by foundational ontologies improves the interoperability of Drammar with other domain ontologies that share the same foundations.

- A bottom up, systematic examination of dramatic elements and their axiomatization in a coherent whole. This process is driven by the description of the elements of drama provided in the previous section, expressed in the form of uncontroversial assertions about each element.

We employ two types of conceptual tools to bridge the gap between the top-down and the bottom-up approaches described above:

- A set of theories, mainly derived from Artificial Intelligence, provide the terminology for describing dramatic elements in a way that is consistent with the high-level categories of foundational ontologies. In particular, we borrowed the model of agent from the theories of rationality put forth by Bratman (1987) and Dennett (1987), since these theories are adequate to express the notion of character in drama illustrated in Section 2, and, at the same time, they are accounted for by foundational ontologies (Masolo et al., 2002). Similarly, the account of drama incidents relies on the theories proposed by AI to model action and change (McCarthy, 1986; Shoham, 1987). More generally, a relevant background for the formal definition and representation of drama is provided by the research in the representation of narratives conducted by Artificial Intelligence since the 1970s: although this research does not specifically address drama, it provides some theoretical and representational tools for an ontology of drama, such as scripts (Schank and Abelson, 1975) and frames (Minsky, 1977).

- A set of modelling devices, which provide the representational tools for mediating between the high-level categories and the specific elements of drama. This is the case for data structures, which are explicitly modeled in Drammar by adhering to the standard data structure definitions available 
in computer science. For example, the tree data model was employed to formalize the notion of recursive structure of drama. At a higher level of abstraction, design patterns (Gangemi, 2005) have also been employed to model the complex interplay of the entities in the ontology, for example in the description of actions.

The theoretical apparatus of Artificial Intelligence has provided a relevant background to the definition of the notion of agency in drama. In order to represent the notion of character (agent in Drammar terms), and to account for the connection between the mental states of the characters and their actions, we relied on Bratman's theory of bounded rationality (Bratman, 1987), since it explicitly ties action to intention. This theory, inspired by the notion of "intentional stance" proposed by Dennett (1987), offers a widely accepted account of how agents' mental states determine their behavior, thus providing the causal connection between characters' mental states and actions that characterizes the definition of action in drama. A formal account of the theory of bounded rationality is provided by the so-called Belief-Desire-Intention (BDI) model (Bratman, 1987; Bratman et al., 1988; Rao and Georgeff, 1991).

According to this model, an agent is a tripartite function of beliefs, desires (or goals), and intentions, where the beliefs are the knowledge of the agent (what she/he knows or believes to be true about the world and about her/himself), and goals are the objectives to be achieved through practical intentions, often referred to as action plans. The description of characters in Drammar is then inspired by the BDI model, i.e., characters are represented as having goals and executing plans to achieve them. For example, consider the Third Act of Shakespeare's Hamlet, where Ophelia meets Hamlet with the motivation of giving him back his presents. In this scene, Ophelia's real goal (that she has assumed from Claudius and Polonius) is to ask Hamlet about his inner feelings, as a way to reveal his madness; in response to her initiative, Hamlet tells Ophelia to go to a nunnery, as a way to achieve his goal to save her from the corrupted court of Elsinore. Later in the scene, Hamlet's and Ophelia's incompatible goals give rise to a dramatic climax as this opposition becomes apparent.

Within the framework described above, characters' plans play a central role, although they are only indirectly perceivable in drama through the actions that the characters execute as part of their plans. In agent theories, plans wrap actions into sequences finalized to achieve goals, giving coherence to the agent's behavior. Following a well established tradition in AI, Drammar represents the plans of the characters according to the STRIPS-like format proposed by Fikes and Nilsson (1971): a plan is formed by a sequence of actions and has preconditions and effects. The different granularity of units in the hierarchical structure of drama is accounted for in Drammar through the paradigm of hierarchical planning (Nau et al., 1999; Sardina et al., 2006), according to which a plan can encompass not only actions but also other simpler plans. So, for example, Ophelia's plan to induce Hamlet to confess his inner feelings can be broken down into simpler plans and actions, including steps such as getting to meet Hamlet and talking to him, each characterized by a specific set of preconditions (being in same room, having the interlocutor's attention, etc.). Plans also give visibility to the notion of conflict, letting the opposition of agents' goals emerge at the level of visible actions. Agent models represent conflicts over plans as the impossibility to carry out two or more actions belonging to different plans - since one obstructs the other, and vice versa (van Riemsdijk et al., 2009). For instance, Ophelia's goal to induce Hamlet to talk about his inner feelings raises a conflict when, as part of her plan to achieve this goal, she lies to him, and her lie obstructs Hamlet's plan to save her, believed to be honest, from the corruption of the court.

As a dynamic, temporal medium, drama unfolds through a sequence of states that occur as a consequence of the characters' actions, bringing about relevant changes in the drama world, and in characters' minds. In order to model the role of characters' intentions in determining the way the world evolves in 
drama, Drammar refers to the logical accounts of world dynamics, such as Situation Calculus theorized by McCarthy (1986). In Situation Calculus, actions work as functions that, when applied to a situation, or world state, change it into another; so, the evolution of the world can be represented as a sequence of states interleaved by actions. The notion of preconditions and effects in Drammar is employed to substantiate the interaction of an agent with the environment, since the agent, by executing practical actions, affects the world state through the changes it determines with its actions. Following the paradigm of Situation Calculus, in Drammar, the evolution of drama is modeled through the notion of a timeline, i.e., a sequence of one or more actions, executed by different characters and encapsulated into units, that bridges the world from a state to another. For example, consider again Hamlet's Third Act, where Hamlet, as a way to test Ophelia, asks her where her father, Polonius, is: Ophelia's answer "At home, my Lord" to Hamlet's question "Where's your father?" brings the drama to a new state where Hamlet clearly knows that Ophelia is lying and that she has been corrupted by the obedience to her father.

Last but not least, the design of Drammar had to deal with the representation of world knowledge: when encoding dramatic works, it is necessary to describe the incidents occurring in them and the participating entities in terms of their real world counterparts. For example, an action of giving can be performed quickly or slowly, an agent can be a human or an extraterrestrial, a pencil can be red or blue, and so on. This kind of knowledge, however, being not specific to drama but pertaining to general knowledge about the world and its linguistic description, is not to be incorporated in the ontology of drama. As a precondition to the representation of drama incidents, Drammar borrows the distinction between perdurants and endurants entities from DOLCE (Descriptive Ontology for Linguistic and Cognitive Engineering (Masolo et al., 2002)). Because DOLCE is committed to a cognitive and linguistic perspective on foundational issues of ontology, it provides a conceptual framework specifically oriented to the description of human activities and the development of applications targeted to human users. In DOLCE, the distinction between endurants and perdurants is based on the behavior of entities with respect to time: endurants are wholly present at any time during their existence, while perdurants are only partially present at any time they are present. In Drammar, perdurants form the temporal dynamics of drama (intentional actions and naturally occurring processes); endurants (characters and objects) take part in them with various roles.

Adopting a role-based representation of the participation of endurants (characters and objects) to incidents, however, is only preliminary to the description of the type and quality of the incidents and of their participants. In order to acknowledge the facts, places, conventions, etc. referred to by actual dramatic works, we chose to incorporate into Drammar an interface to external semantic and lexical resources, leaving to the single annotation initiative the task of locating these resources externally to Drammar. The choice of the semantic and linguistic resources to be referred can change, provided they are compliant with the role-based representation of incidents assumed by Drammar. The drama annotation tool described by Cataldi et al. (2013) provides an example of this approach. This tool, developed in parallel with Drammar, implements a meaning negotiation process: the annotator looks up the (English, Italian, etc.) word describing the incident to be annotated in MultiWordNet (Pianta et al., 2002); by relying on the mapping with WordNet (Miller, 1995), the tool retrieves the relevant concepts from the SUMO ontology (Pease et al., 2002), leaving to the annotator the task of selecting the appropriate concept.

\section{Ontology design}

Most ontology engineering methodologies are geared to environments characterized by production or management processes of some type, which must be standardized and regulated with the help of 
the knowledge encoded in the ontology. The input to these methodologies consists of structured documentation and data, often expressed in some digital format, accompanied by specific professional roles; critical issues typically stem from the integration of the ontology-based approach into the existing management processes and from the definition of the ontology maintenance processes, as discussed by Sure et al. (2004) and Suarez-Figueroa et al. (2012). With the advent of distributed frameworks in knowledge representation such as Linked Data, ontology engineering has shifted towards an approach characterized by interdisciplinarity and fragmentation, managed through cooperative frameworks for the design of ontologies (Simperl and Luczak-Rösch, 2014; Noy et al., 2013). Partly in contrast with this trend, Drammar aims at constituting an authoritative model, carefully crafted through a centralized approach, despite the fragmentation of the sources by which it is inspired - which include the bulk of work in drama literature through the ages, and the multi-disciplinary nature of the representation of drama. From this perspective, the NeOn ontology engineering methodology (Suarez-Figueroa et al., 2012) is the most suitable for grasping the peculiarities of the development of Drammar, thanks to its flexibility and its focus on the relation of the ontology with non-ontological resources, such as linguistic and other semantic resources. In the following, after describing the specific challenges posed by the representation of drama for ontology engineering (Section 4.1), we describe how these challenges were addressed within the conceptual framework provided by $\mathrm{NeOn}$ (Section 4.2).

\subsection{Encoding the domain of drama: The issues}

Given the background described in Section 2, the task of expressing knowledge about drama through an ontology poses a set of specific challenges for ontology engineering:

1. Drama as a cultural object. The domain of drama mainly refers to the features of a cultural object (namely, drama) that can be found in a multiplicity of forms, all sharing a basic set of features. Differently from most domains encoded in formal ontologies, which center on the normative description of process types, here processes are embedded - as plot incidents - into the extremely variable manifestations of drama, reflecting the entire range of human activities, with no limitation on the representable types of processes other than cultural factors.

2. Instantiated drama description. Due to the mimetic nature of drama, a large part of the data about drama can be seen either as characters' actions, or "instructions of acting", according to the definition proposed by Elam (1980), that need some form of linguistic description and/or a classification with respect to the commonsense knowledge situated beyond the boundaries of drama definition. At the same time, the ontology must be able to express a definition of the dramatic qualities that apply to all their diverse manifestations in a variety of languages and media.

3. Multiple tasks. The representation of drama is shared by a set of different tasks, which differ in goals and procedures and are carried out by different professional roles. Consider, for example, drama production, aimed at artistic expression and carried out by well coded roles such as directors and actors, and drama preservation, aimed at encoding in digital form a dramatic work (realized through different media and languages), carried out by interdisciplinary teams including drama critics, media experts and scholars in digital humanities.

In order to face the challenges posed by the drama domain, the design and implementation of the ontology of drama required the elaboration of an original methodology. We refer to the NeOn toolkit (Suarez-Figueroa et al., 2012), since this methodology, structured as a highly flexible set of scenarios, better suits the specific challenges posed by the drama definition and representation. 
Notice that the specific challenges posed by the drama domain have led the design of Drammar to follow a principle of minimality: targeted at representing the core elements of drama, and not geared to specific tasks, Drammar relies on external sources to encode the commonsense and linguistic knowledge in instantiated drama. However, given the multiplicity of these manifestations and the variability of tasks, we did not make commitments to specific resources in the ontology design, since this would be in contrast with the need to keep the ontology task- and media-independent; instead, we developed a mapping towards external sources, that we illustrate in Section 5.

\subsection{Drammar development in the framework of the NeOn methodology}

The NeOn ontology engineering methodology (Suarez-Figueroa et al., 2012) consists of a set of scenarios for the development of ontologies in a collaborative way. Also, $\mathrm{NeOn}$ introduces the notion of "reference community", characterized by needs and goals that drive the ontology development. This feature is particularly relevant for the drama domain, given the well specified, but heterogeneous, set of tasks and roles that benefit from the formalization of drama. Briefly, the NeOn methodology maps a set of activities onto 9 scenarios for building and maintaining ontologies and ontology networks. Activities (and the larger processes they are part of) are formally defined in a glossary of 59 entries. As an example of a process, consider Ontology aligning, which may involve activities such as Ontology mapping and merging. The set of scenarios envisaged by $\mathrm{NeOn}$ is not prescriptive: depending on the characteristics of the single ontology engineering project, only some scenarios may be relevant to that project.

Table 1 illustrates the mapping of Drammar onto NeOn scenarios. In line with the spirit of $\mathrm{NeOn}$ only some scenarios and activities specifically apply to the design and development of Drammar. In particular, Scenario 1 (From Specification to Implementation), Scenario 2 (Reusing and Re-engineering

The mapping of the design and development of Drammar onto NeOn scenarios and activities (from Suarez-Figueroa et al. (2012))

\begin{tabular}{|c|c|c|}
\hline NeOn Scenario & Activities & Drammar \\
\hline \multirow[t]{4}{*}{$\begin{array}{l}\text { 1. From Specification to } \\
\text { Implementation }\end{array}$} & $\begin{array}{l}\text { 1. Ontology requirements } \\
\text { specification }\end{array}$ & $\begin{array}{l}\text { Identification of the elements of drama and translation } \\
\text { into protoaxioms }\end{array}$ \\
\hline & 2. Scheduling & Identification of the priorities (core elements) \\
\hline & 3. Ontology formalization & Translation of the proto-axioms into axioms \\
\hline & 4. Implementation & Implementation in OWL2 \\
\hline \multirow[t]{3}{*}{$\begin{array}{l}\text { 2. Reusing and Re-engineering } \\
\text { Non-ontological Resources }\end{array}$} & $\begin{array}{l}\text { 1. Searching non-ontological } \\
\text { resources }\end{array}$ & Survey of the linguistic and commonsense resources \\
\hline & $\begin{array}{l}\text { Assessing the candidate } \\
\text { non-ontological resources }\end{array}$ & $\begin{array}{l}\text { Evaluation of the selected resources through annotation } \\
\text { case studies }\end{array}$ \\
\hline & $\begin{array}{l}\text { 3. Selecting the most appropriate } \\
\text { nonontological resources }\end{array}$ & $\begin{array}{l}\text { Selection of the resources (WordNet/MultiWordNet, } \\
\text { YAGO2 and FrameNet) }\end{array}$ \\
\hline \multirow[t]{2}{*}{$\begin{array}{l}\text { 5. Reusing and Merging } \\
\text { Ontological Resources }\end{array}$} & 1. Alignment & $\begin{array}{l}\text { Partial alignment with DOLCE (endurant/perdurant } \\
\text { distinction) and OLO (Ordered List Ontology) }\end{array}$ \\
\hline & 2. Merging & Merging of the classes/properties into Drammar \\
\hline $\begin{array}{l}\text { 7. Reusing Ontology Design } \\
\text { Patterns }\end{array}$ & & $\begin{array}{l}\text { Re-use of the Situation Description and Role design } \\
\text { patterns }\end{array}$ \\
\hline
\end{tabular}


Non-ontological Resources), Scenario 5 (Reusing and Merging Ontological Resources) and Scenario 7 (Reusing Ontology Design Patterns) are relevant for Drammar.

Scenario 1 (From Specification to Implementation), encompasses four activities: ontology requirements specification, scheduling, ontology formalization, and implementation. The ontology requirements specification phase was led by a drama expert, in charge of distilling from the literature a coherent and consistent set of requirements for the ontology, as described in Section 5.2. The output of this phase consisted in a set of core elements to be primarily included in the representation of drama. Basic criteria for inclusions were the importance and continuity of these elements in the literature. Other criteria were provided by the identification of the tasks the ontology would address, from conceptual modelling and metadata annotation, to drama preservation, drama analysis, and drama generation. The output expected from this phase, the Ontology Requirements Specification Document (ORSD), consisted in the identification of the elements of drama, accompanied by the references to the literature that motivate and support the definition of each element. For each element, a description was provided both as informal free text (illustrated in Section 2) and as a collection of semi-formal statements specifying the nature of the element (Section 5.2).

In Scenario 2 (Reusing and Re-engineering Non-ontological Resources), non-ontological resources (such as classification schemes, thesauri, lexica, etc. characterized by having achieved some level of consensus) are selected and possibly re-engineered for the integration into the ontology, based on the requirements that the ontology should fulfill. In accordance with the prescribed activities for this scenario (Searching non-ontological resources, Assessing the candidate non-ontological resources and Selecting the most appropriate ontological resources), the available resources were surveyed and selected by taking into account the requirements for drama description: in particular, given the focus on the representation of incidents in the annotation of drama, highly structured linguistic resources for the representation of processes and events were given priority.

As discussed in Section 3, the description of media objects goes beyond the conceptual model of drama and requires the specific entities displayed in an object (characters, incidents, places, etc.) to be described based on linguistic and semantic representation systems situated externally to the conceptual model of drama. The survey also accounted for the requirements posed by Challenge 3 (Multiple tasks), i.e., the need to gear the ontology design to different tasks. Based on the survey, we selected a set of non-ontological semantic resources, such as lexical-semantic resources, to cope with the linguistic counterparts of drama elements, and we developed an interface with these resources: WordNet and MultiWordNet (Miller, 1995; Pianta et al., 2002) for the description of incidents and entities, and FrameNet (Baker et al., 1998), for the argument structure of incidents. Thanks to their interoperability, these linguistic resources are open to the use of commonsense and world knowledge sources (such as YAGO2 (Hoffart et al., 2013) or DBpedia (Bizer et al., 2009)) according to project-specific choices. As for the re-engineering activities encompassed in this scenario, they mostly concerned the implementation of the interface with the aforementioned resources as a module of the ontology (Cataldi et al., 2013), an activity termed Ontology forward re-engineering in $\mathrm{NeOn}$ (see the description of the ExternalReference class in Section 5.1).

Scenario 5, Reusing and Merging Ontological Resources, is broken down, in NeOn, into the activities of alignment and merging. In Drammar, the hierarchy of classes was influenced by the philosophical distinction between perdurants and endurants in DOLCE, as anticipated in Section 3. For this reason, the taxonomy includes the classes needed to model this distinction, reflected also in the modelling of processes, which were aligned with some specific classes in DOLCE (see Section 5). This process also concerned the Ordered List Ontology (Abdallah and Ferris, 2010), necessary to represent the sequential 
nature of agents' plans. Finally, as the design of the ontology proceeded, its taxonomy was checked against the requirements posed by the ontology verification methodology termed OntoClean, described by Guarino and Welty (2009); this verification was primarily conducted in order to keep the representations of entities and roles well distinct in the ontology.

Scenario 7 Reusing Ontology Design Patterns involved the re-use of design patterns for the representation of drama incidents (Gangemi, 2005): their description is inspired by the specific pattern for the representation of tasks according to a role-based template. Here, due to the goal of minimality stated before, a formal alignment was not conducted, in order to reduce the technical apparatus of the ontology.

Concerning the life cycle of the ontology, after the completion of the core ontology, the development of Drammar was affected by some subsequent research tasks. Undertaken for studying some specific aspects of drama, in some cases they led to minor re-design phases. In terms of the NeOn methodology, this corresponds to the Iterative-Incremental Ontology Network Life Cycle Model, according to which an initial set of requirements is translated into a formal ontology by checking it against practice and adding new components as the development continues. For example, the development of a tool for drama visualization (Lombardo and Pizzo, 2016) allowed for the detection of some gaps and incorrect modelling choices, with consequent re-design: in particular, it prompted some modifications to the notion of plan, which was enriched with information about plan achievement. The achievement of plans, in fact, turned out to be relevant for tracking the characters' intentions over drama through failures and replanning. In parallel with the redesign and refinement process, the core model of drama encoded in Drammar provided a basis for ad hoc extensions tailored to specific tasks and theories. For example, in order to annotate the emotions of the characters in a semi-automatic fashion, an extension of the ontology for the representation of affect was implemented. This extension, based on the cognitive model of emotions by Ortony et al. (1988), was coupled with a rule-based module that computes characters' emotions in response to the incidents occurring in drama (Lombardo et al., 2015a,b).

\section{The ontology}

In this section, we first describe the taxonomy of the classes in Drammar (Section 5.1), then we illustrate the patterns of classes and relations that we use to represent specific drama works (Section 5.2). ${ }^{5}$ Informed with the conceptual foundations for drama representation introduced in Section 3, the taxonomy connects the foundational bases with the drama elements, which form the leaves of the hierarchy.

\subsection{A taxonomy for drama representation}

The design of the taxonomy follows the well known principle according to which a class is specialized into subclasses depending on the value of a specific trait. Each trait/property accounts for a specific aspect of drama and its position in the taxonomy marks its importance, with the most general distinctions set at the higher levels in the taxonomy and the most specific distinctions at the lower levels. As an example of the taxonomy design, consider the class action: the concept of action is defined as the sum of the traits accumulated top-down along the taxonomy: temporal nature, for being a type of DramaPerdurant (as opposed to DramaEndurants, which are independent of time), accomplishment, for being a (telic) process (as opposed to states), and intentionality, for being an action (as opposed to non-intentional events).

\footnotetext{
${ }^{5}$ The ontology can be found at http://purl.org/drammar.
} 
The top-level of Drammar contains four classes:

- DramaEntity is the class of the dramatic entities, i.e the entities that are peculiar to drama;

- Datastructure is the class that organises the elements of the ontology into common structures (namely, list, sets and trees);

- DescriptionTemplate contains the patterns for the representation of instantiated drama according to role-based templates;

- ExternalReference is the class that bridges the description of drama to commonsense and linguistic concepts situated in external resources.

The design of the top-level classes is also motivated by the need to avoid the subsumption violations that constitute the object of the OntoClean methodology (Guarino and Welty, 2009), such as the antirigid concepts of role subsuming the rigid concept of agent. So, the three types of entities mentioned above, namely drama entities, roles, and data structures, were kept separated, forming disjoint subhierarchies in the taxonomy.

\section{DramaEntity}

DramaEntity groups all the elements that belong to the drama domain. It is divided into two subclasses, each describing specific drama elements:

- DramaPerdurant represents the processes that occur in drama. DramaPerdurant corresponds to the class Perdurant in DOLCE (rdfs: subclassof of the class perdurant in DOLCE-Lite, ${ }^{6}$ as described in (Masolo et al., 2002)). This class subsumes the elements of drama that, interleaved, describe the advancement of drama, namely processes and states.

- DramaEndurant represents the entities that participate into processes and states. DramaEndurant corresponds to the class Endurant in DOLCE: "Endurants are wholly present (i.e., all their proper parts are present) at any time they are present" (Masolo et al., 2002) (as such, it is formally declared in Drammar as an rdfs: subclassof of the class endurant in DOLCE-LITE). They are agents and objects, kept distinct from each other by the feature of intentionality: agents intentionally perform actions, while objects are simply involved in the actions.

The DramaPerdurant class divides into:

1. the Process class.

2. the State class.

Both processes and states play a two-fold function, since they are related with both drama incidents (i.e., the perceivable dynamics of drama) and characters' plans (the non visible, inferable motivations of characters). Processes and states have been declared, respectively, subclasses of the classes of the same name in DOLCE-LITE. Encapsulated in units, processes are the basic constituents of timelines, i.e. sequences of units occurring over the course of the drama. Intentional processes (or actions) are also the basic elements of characters' plans. The term process in Drammar is employed as a synonym of the term event in DOLCE, and includes both events and processes in DOLCE, blurring the distinction between non-telic (statives) and telic processes (accomplishments and achievements) acknowledged by DOLCE, which is not relevant in drama. ${ }^{7}$ Similarly to processes, states are also related with both drama

${ }^{6}$ http://www.loa.istc.cnr.it/ontologies/DOLCE-Lite.owl

${ }^{7}$ Although non-telic actions can be put on stage in drama (such as a passer-by crossing the street in a movie clip), they are not relevant for drama construction since they do not contribute to the dramatic qualities of the work, similarly to many other details of the mise-en-scène. 
incidents and characters' plans: interleaved with timelines, states form the dynamics of drama; as part of plans, states form their preconditions and effects. Both types of perdurants are described by role-based templates, located below the DescriptionTemplate top level class (see below).

The State class further divides according to the entity type to which the state is attributed. The entity of attribution can be agent, action or world, thus yielding two subclasses:

1. Mentalstate for subjective states attributed to agents;

2. StateofAffairs for objective states related with plans and timelines.

Inspired by Ferrario and Oltramari (2004), mental states are the core of the description of the agents, providing motivations for their behavior. The subclasses of the Mentalstate class, then, are based on propositional attitude types, and acknowledge also the rational vs. irrational distinction with the inclusion of emotions:

- Belief: the agent's subjective view of the world;

- Goal: the objectives that motivate the actions of the agents;

- Emotion: the emotions felt by the agent;

- Value: the moral values acknowledged by an agent, which are engaged by the unfolding of the plot.

The DramaEndurant class subsumes the entities participating in the drama incidents (modeled via the notion of perdurant, as illustrated above). Similarly to the DramaPerdurant class, this class also divides into more specific classes:

1. Agent represents the entities that intentionally act in the drama incidents, usually named characters.

2. Object represents the unintentional entities that participate in the incidents such as locations and physical objects (often called "props" in drama production).

The Agent and Object classes are disjoint, since the intentional trait is rigid and cannot be acquired or lost within a given drama. The characterization of agency as intentional is also in line with DOLCE, which encompasses agentive physical agents as a type of physical objects (a type of endurants in the taxonomy of DOLCE). Notice that, in drama, an entity type which is considered non-intentional in commonsense knowledge, such as, e.g., a volcano, may become an intentional agent for dramatic reasons: this is precisely why the intentional/non-intentional trait is modeled in the drama domain, instead of delegating it to external categories. ${ }^{8}$ The instances of the DramaEndurant class (defined as the union of agents and objects) play roles in processes and states, and are the fillers of the role-based linguistic templates describing states and processes. As characterized by intentionality, agents are those entities in drama that potentially:

- have goals;

- intend plans to achieve their goals.

Also, agents (i.e., instances of class Agent in Drammar) are involved in actions by playing some role (i.e., being the filler of the role) in frame of the action (see DescriptionTemplate class below).

\footnotetext{
${ }^{8}$ Notice also that the characterization of an entity as an intentional agent does not prevent this entity from playing a role in unintentional processes; in other words, agency is kept separated from the intentionality trait of processes, so as to allow for more flexible configurations, such as an agent unwillingly causing a process to start (e.g., letting an object fall off a table).
}

\section{1}




\section{DataStructure}

The class DataStructure encodes the structures that superimpose an organization onto the elements of drama. Remember from Section 2 that the peculiar organization of drama is itself a core element of drama. The class DataStructure includes abstract data types (subsumed by the AbstractDataType class), such as lists or sets, and their components (subsumed by the class of abstract data type components, ADTComponent class), such as list elements or set members. In Drammar, data types provide containers for grouping the elements of instantiated drama according to the acknowledged structural elements that characterize the organization of dramatic action, such as scenes and plans. The data structure types are characterized by the features of being ordered, unordered or hierarchical. So, the DataStructure class includes sequential structures (List class), whose elements are ordered; set structures (Set class), which group elements of the same type but lack an internal ordering of the elements; hierarchical structures (Tree class). With respect to their consequences for modelling drama, data structures are defined in the following way:

- List is inspired by a well known ontology, the Ordered List Ontology ${ }^{9}$ ( List is a subclass of the Ordered List class in OLO): drammar:List rdfs:subclassof olo:OrderedList). The type of drama data contained by the list elements (instances of class OrderedListelement) identifies the specific type of list: for example, plans (class Plan) are lists of simpler plans (AbstractPlan class) or actions (DirectlyExecutablePlan class); timelines (class Timeline) are lists of units. Precedence relations are encoded for the list elements, also marking the first and the last elements of a list, respectively.

- Set gathers unordered elements of the same type (instances of the SetMember class): in drama, this is the case of units (Unit class), i.e., sets of processes which compose the timelines, and of state sets, which provide the preconditions and effects of timelines and plans, respectively. State sets can be internally consistent (ConsistentStateSet), or can include conflicting elements (ConflictSet). The first type provides the preconditions and effects of timeline, which are necessarily internally consistent, while the latter serves the function of modelling the conflicts which may arise from the intentions (i.e., plans in Drammar) of different characters.

- Tree represents tree-like structures. In drama, tree-like structures are needed to represent the notion of scene: a scene, of larger or smaller granularity, can subsume other scenes, and can be subsumed by larger scenes. A tree contains instances of the TreeNode class, a type of ADTComponent characterized by the parent-child relations over them. A Scene, then, is a type of TreeNode which is characterized by a relation with the temporal organization of drama into timelines.

\section{ExternalReference}

The class ExternalReference bridges the representation of drama onto commonsense and linguistic concepts stored in external resources. This class and its subclasses provide the interface between the elements of drama and the external knowledge sources needed to describe instantiated drama (e.g., for describing type and qualities of actions and agents), but they are not relevant for representing the structural components of drama and the relations over them. In Drammar, no linguistic or commonsense information is directly attached to any drama entity: by doing so, in case the external reference system varies, only the properties of the class ExternalReference and its subclasses are affected, while the structural representation of drama remains unchanged, and can be exchanged across systems that

\footnotetext{
${ }^{9}$ http://purl.org/ontology/olo/core
} 
rely on different external knowledge sources. This class divides into subclasses which bridge specific drama elements onto their linguistic and semantic counterparts:

- ExternalRefEntity maps a perdurant (process or state) or an endurant (agent or object) onto its description: for instance, the mapping target may be the identifier of a lexical entry for describing a process (for example, the term "kill" in WordNet), or the IRI of an ontology class for describing an object (for example, the concept of "Weapon" in SUMO).

- ExternalRefSchema maps a process or state onto a verbal frame that describes it according to a role structure, with the class ExternalRefRole to map the single roles onto their description in the frame (for example, the frame for "Killing" in FrameNet (Baker et al., 1998)). Notice that, despite the implementation of a specific class for interfacing the representation of instantiated drama with FrameNet, the role-based model encoded in Drammar is neutral with respect to the specific resource and could be fit to other resources that acknowledge the same schema, including projectspecific repositories.

- ExternalRefEmotionType maps the emotions of the characters onto a reference model of emotions, semantically described outside the boundaries of Drammar (for example, the emotion of Fear in the model by Ortony et al. (1988)).

\section{DescriptionTemplate}

The class DescriptionTemplate contains the patterns for encoding the role-based schemata. It has the purpose of mapping a situation (as intended by Gangemi and Presutti (2009)), be it a process or state, onto its linguistic description. Its subclasses, namely Role and SituationSchema, provide the primitives to realize a role schema for describing a situation. The SituationSchema class represents the description of a situation in terms of the roles involved in it (see the Situation Description ontology pattern (Gangemi and Presutti, 2009)). In order to map the entities (agent and objects) involved in the situation onto specific roles, this class is related to the Role class through specific properties.

Class SituationSchema divides into subclasses for representing specific schema types:

- FramenetSchema, for mapping the description of entities onto the linguistic reality encoded in lexical-semantic resources, e.g., FrameNet (Baker et al., 1998).

- MentalstateSchema, for mapping the description of a mental state onto specific schemata for the different types of mental states, each of which is committed to a specific model: for example, the ValueSchema relates an agent's value engaged in a given timeline or plan (Value) with some reference value system (which may be shared by agents). The MentalstateSchema further specifies into BeliefSchema, EmotionSchema, GoalSchema, ValueSchema.

\subsection{From statements about drama to drama patterns}

In this section, we describe how the drama elements put forth in Section 2 are translated into patterns of classes and properties for representing the elements of drama: action, agent, conflict and structure. For each element, we summarize its definition through a list of semi-formal statements. For the sake of readability, the ontology axioms have been numbered and gathered in Appendix A. In figures, some graphical simplifications have been adopted for the representation of sets and lists, as illustrated in Appendix B; also, we have established the following conventions for the colors (with the exception of Fig. 4, where colors mark the structural elements of drama): the terminal elements of the taxonomy (e.g., agent or action) are represented by white boxes, while non-terminal elements are represented by gray boxes; dark gray boxes represent the subclasses of the DescriptionTemplate class. 


\subsubsection{Action}

From the literature (Aristotle, 2013; Olson, 1961; Polti, 1895; Spencer, 2002; Szondi, 1983) we have distilled the following statements:

(A1) Action is the foundational element of drama.

(A2) Actions are organized according to a logic of cause and effect.

(A3) Action is motivated by a character's goal.

(A4) Action is part of a character behavior that has some degree of unity and wholeness.

(A5) Action is the enactment of a character's deliberation.

(A6) Action defines the character function in the plot, therefore the characters are defined through their actions.

(A7) Action is a scalable notion: the drama is an action that can be discretized into increasingly smaller chunks.

Figure 1 provides a schematic overview of the representation of actions. As a constituent of both plans and timelines, action provides the connection between the intentional architecture of drama and its observable manifestation.

Since action is "the foundational element of drama" (Statement A1), we encode action as:

- A class of the ontology (Axiom 1);

- An element that is necessarily contained in any dramatic structure, at any level of granularity: actions are gathered into units (Axiom 2), which have their position in a timeline (Axiom 3)

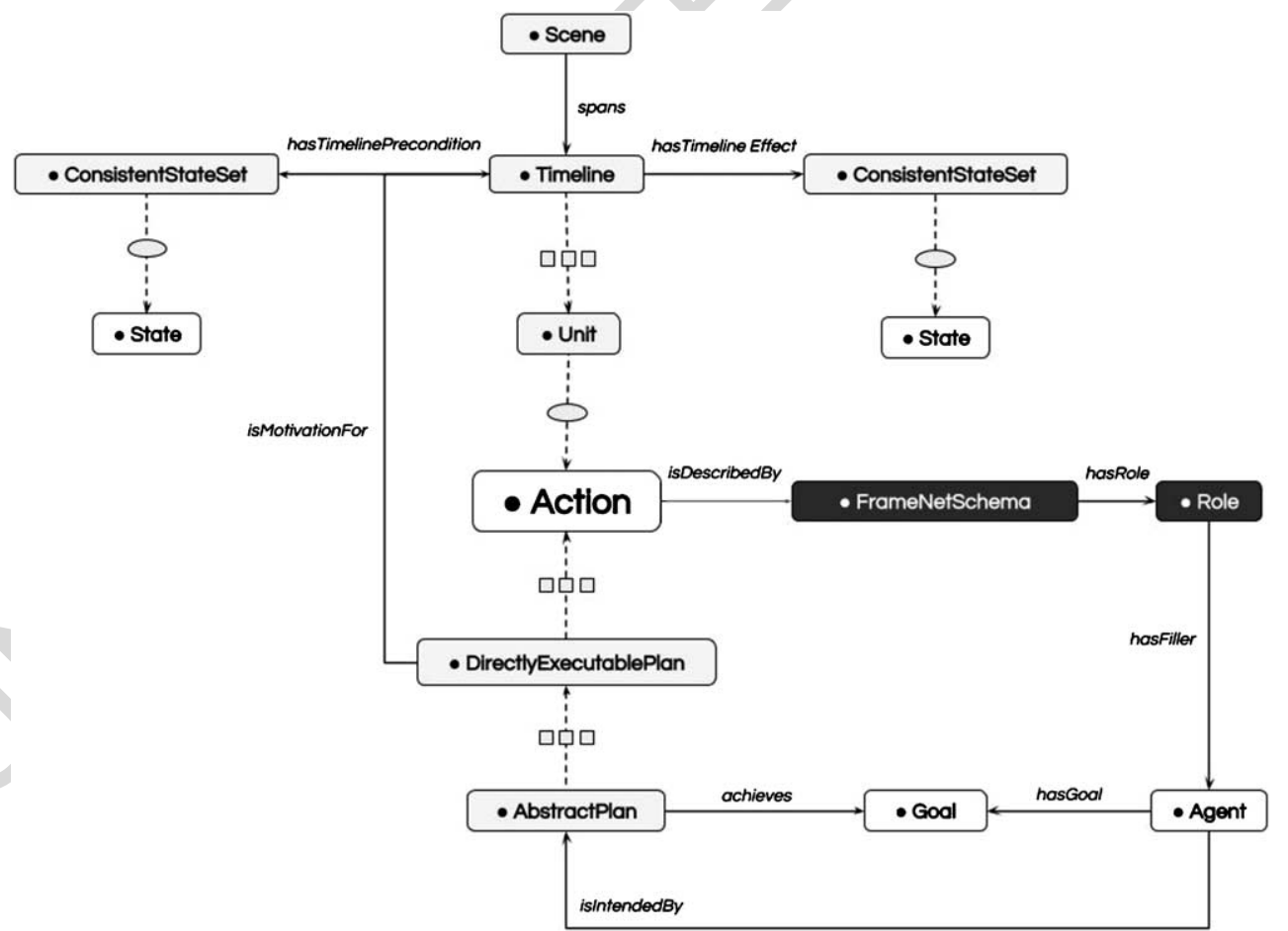

Fig. 1. The pattern for actions Drammar. The oval-shaped and list-shaped symbols on the edges represent, respectively, the patterns for sets and lists (see Figs 7 and 8 in Appendix B). 
spanned by some scene (Axiom 14) - the fundamental dramatic structure (Fig. 1, upper part, middle).

- The basic elements of recursive plans (Axioms 4 to 6), which connect agent's high level goals with practical, directly executable actions (Fig. 1, lower part, middle).

The organization of actions "according to a logic of cause and effect" (Statement A2) is realized by connecting actions to their precondition and effects (set of states in Drammar); since chains of actions can lead to complex effects, not accounted for by single actions, actions have been generalized into units, sequenced into timelines. So, preconditions and effects are not attached to actions or units, but to timelines (via the hasTimelinePrecondition and hasTimelineEffect properties, see top of Fig. 1); they are intended as the states that hold, respectively, before and after the occurrence of the incidents in the timeline.

Statement A3, "action is motivated by a character's goal", also requires generalizing actions to higher level structures. As displayed in the lower part of Fig. 1, actions are the elements of directly executable plans. Abstract plans contain actions indirectly, through the recursive containment of other abstract plans and eventually of some directly executable plans. An action is motivated by an agent's goal through the plan which contains it, and which is directly connected to the goal. ${ }^{10}$

Strongly connected to Statement A3 are Statements A4 and A5. Statement A4, "actions are part of a character's behavior that has some degree of unity and wholeness", refers to the relationship of the single action with the larger context of the whole drama. It is the hierarchy of plans that implements the unity and wholeness mentioned in the statement, since actions are related to abstract, long-term plans by climbing the hierarchy.

Statement A5, "action is the enactment of a character's deliberation", is realized through the alignment of the actions observed in a drama with the plans of the characters, represented by the property isMotivationFor, which connects a plan with a timeline (see the vertical arc from Plan to Timeline in the left part of Fig. 1). So, each action in the timeline is motivated by some (possibly different) plan, meaning that each action results from a structured deliberation process, and each plan eventually motivates an observable sequence of actions (Axioms 5 and 6).

Statement A6, "action defines the character function in the plot, therefore the characters are defined through their actions", acknowledges action as the perceivable element in the drama and the major tool for defining characters. In Drammar, actions are related with agents through the role (class Role) they fill (isfillerof property) in the schema (class ProcessSchema) describing (describes property) an action (see the right part of Fig. 1).

Finally, Statement A7, "action is a scalable notion: the drama is an action that can be discretized into increasingly smaller chunks" has been accounted for in Drammar by interpreting the term action from a two-fold perspective: i) strictly speaking, as the smallest perceivable incident in the drama, licensed by a directly executable plan and embedded in some unit, ii) in a broader sense, as the chunk of the drama licensed by the interplay of characters who play a role in it, encoded into scenes (see the top of Fig. 1).

\footnotetext{
${ }^{10}$ The relation of the plan with goals has not been added as a necessary characteristic to the general Plan class, since there exists, in Drammar, a type of plan which is unrelated to goals, the UnderspecifiedPlan: this type of plan is characterized by the fact that it does not achieve a goal and is empty. Underspecified plans represent the parts of the agent's plans that are not actually implemented in the drama (e.g., due to plan failure or replanning) and, as a consequence, cannot be observed but only conjectured as guessed continuations of abandoned plans.
} 


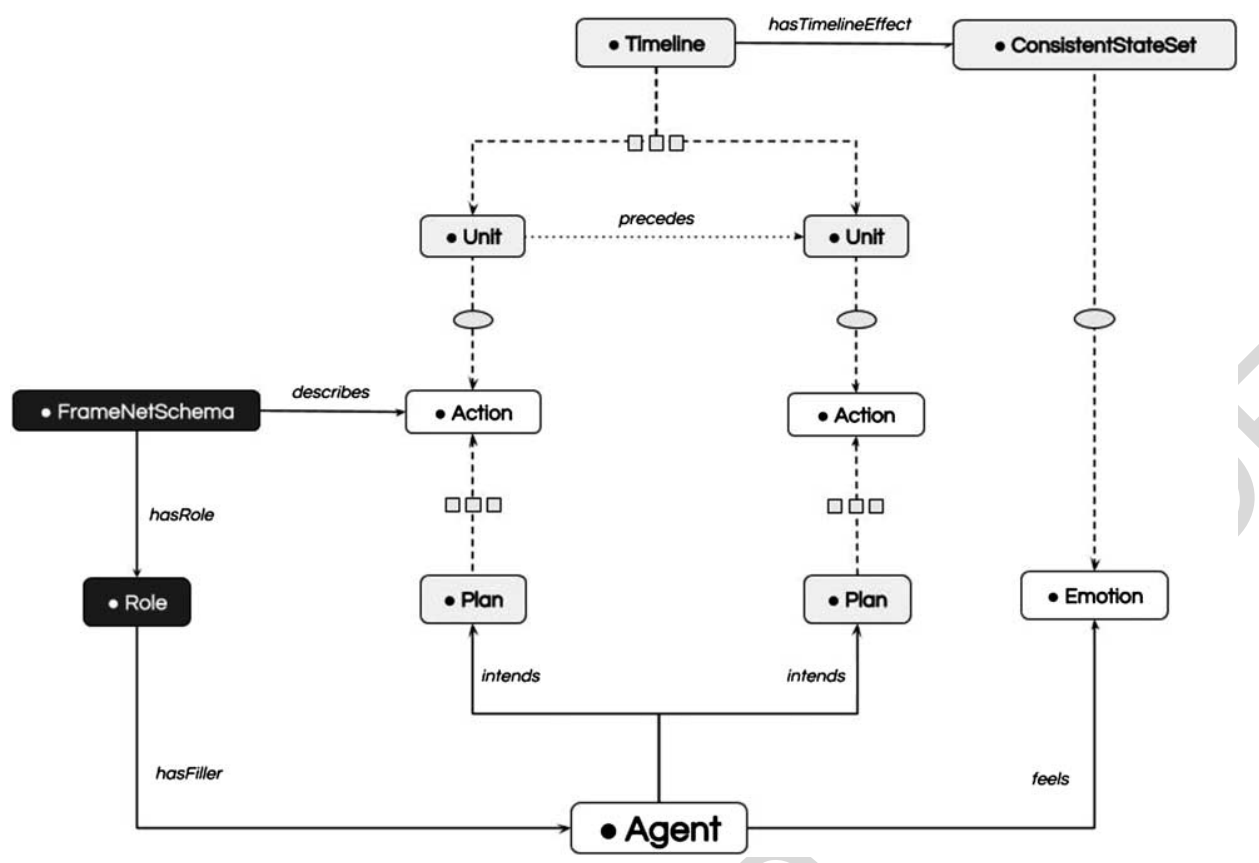

Fig. 2. The pattern for agents in Drammar. See Figs 7 and 8 in Appendix B for the symbols on the edges, representing the patterns for sets and lists.

\subsubsection{Agent}

From the literature (Aristotle, 2013; Hegel, 1885; Egri, 1946; Styan, 1963; Ryngaert, 2008; Currie, 2009; Newman, 2009), we have distilled the following statements:

(AG1) An agent intends at least one action.

(AG2) An agent does at least one action.

(AG3) An agent displays emotions.

(AG4) An agent interacts with the environment.

(AG5) An agent interacts with the other agents.

(AG6) An agent is the medium of representation in drama.

(AG7) An agent starts the action and/or reacts to others' actions.

As the pivotal element of the language of drama, it is the agent's duty to connect the actualized, immanent actions represented in instantiated drama with the intentions underlying them, structured into plans that motivate the actions occurring in the drama and determine the emergence of characters' conflicts. Figure 2 shows the main classes and properties that relate the notion of agent (bottom left) with the notions of action and plan (center of the figure) and timeline (top of the figure).

As described in Section 3, in the BDI model the link between agent and action is provided by the abstract notion of intention, often referred to as "plan" in agent architectures (Bratman et al., 1988; Rao and Georgeff, 1991; Sardina et al., 2006): the agent intends a plan, which contains one or more practical actions that are executed as a consequence of the agent's commitment to that plan. In Drammar, the agent is defined as someone who intends (intends property) a Plan (Axiom 7), which contains actions. This account satisfies the requirement posed by Statement AG1, that "an agent intends at least one action". 
The link between the agent's intentions and the execution of the actions, postulated by Statement AG2 "An agent does at least one action" corresponds to the participation of the agent to the actions represented in drama. Following an established tradition in linguistics and knowledge representation, actions are represented in Drammar by using role-based templates that can be matched onto the thematic roles of verbs in human languages (Fillmore, 1985). The pattern adopted in Drammar specifically assumes the role-based template that characterizes FrameNet (Baker et al., 1998): a FrameNet Schema (subclass of the Process Schema class) is connected to a set of roles (instances of the Role class) by the hasRole property (see the left area of Fig. 1). Each Role, then, is filled by an Agent or Object (subclasses of the DramaEndurant class), with the additional constraint that all the actions gathered in a unit must include at least an agent as role filler. Formally, the ontology constrains at least one of the roles attached to each action to be filled by an agent (Axiom 8). This constraint is in line also with Statement AG5: "an agent interacts with other agents", since the role-based account of actions allows different agents to take part in the same action with different roles. At the same time, it also satisfies Statement AG6, namely that "the agent is the primary medium of representation in drama", since it guarantees the participation of agents to the actions displayed in drama.

As the primary medium of representation, the agent is also the medium by which emotions are displayed in drama (Statement AG3, "an agent displays emotions"): emotions are modeled in Drammar as a type of mental state (i.e., the Emotion class as a subclass of the Mentalstate class, see Axiom A9). The feels property connects the Agent class with the Emotion class, linking each agent to the emotions she/he/it feels. Notice that, in order to acknowledge the plurality of accounts of emotions put forth by the psychological literature, the ontology is neutral with respect to the specific theory employed to describe the emotions of the agents, although it assumes a cognitive account of emotions. ${ }^{11}$

In drama, agents execute actions that affect the world, by changing the world state (Statement AG4, "An agent interacts with the environment"). The changes in the world state brought about by the agent's actions are represented in Drammar by encapsulating actions in units and sequencing into timelines (see the Timeline class, top of Fig. 2), that bridge the world from one state to another. A Timeline, made of action units (Unit class), has preconditions and effects, modeled as sets of consistent states (ConsistentStateset see Axioms 10 and 11).

The behavior of the agents can be both pro-active and reactive: agents both pro-actively devise plans to achieve their goals and react to the other agents' goals that are in conflict with their own goals. This corresponds to Statement AG7, "An agent starts the action and/or reacts to others' actions". The temporal ordering of agents' actions, then, needed to identify the agents' initiative in the action/reaction dynamics, is provided by the notion of timeline. As it can be observed in Fig. 2, an instance of Timeline (top of the figure) lists several units whose actions are part of different Plan instances, possibly intended by different agents.

\subsubsection{Conflict}

From the literature (George Lukács, 1965; Archer, 1912; Brunetière, 1914; Diderot, 1966; Egri, 1946; Field, 2003; Hegel, 1885; Lavandier, 1994; Lessing, 1962; McKee, 1997; Ryngaert, 2008; Spencer, 2002; Sweet, 1993) we have distilled the following statements:

(C1) Conflict is an opposition between agents. The opposition arises from differences in agents' goals and/or values (moral, ethical, political), accountable in their behavior.

\footnotetext{
${ }^{11}$ In previous work, we have chosen to refer to the cognitive theory of emotions described by Ortony et al. (1988), often referred to as the OCC model, for its capability to grasp the emotions of characters demonstrated in annotation initiatives such as that described by Lombardo et al. (2015b).
} 
(C2) Conflict can concern different agents or a single agent. Therefore conflict can be interior or 1 exterior to an agent.

(C3) Conflict is linked to the nucleus of the agent's fundamental psychological traits.

(C4) Conflict provokes an emotional response in the agent.

(C5) Conflict is the contrast between agents and the situation.

(C6) Conflict is represented by an obstacle.

Mainly described as the opposition of characters, conflict is intrinsically related with the characters' minds (goals and values, in Drammar), whose differences emerge through their actions. According to Statement C1 "Conflict is an opposition between agents". The opposition between agents arises from the presence of differences in agents' goals and/or values (moral, ethical, political): however, goals, being mental states, are unobservable entities in drama, which is conveyed to the audience through actions. So, as anticipated in Section 3, in Drammar, the notion of conflict is dealt with at the level of plans, which contain actions. The conflict over plans is defined as their membership in a ConflictSet, a class that gathers conflicting plans in a set (Axiom 12). Agents devise plans to achieve their goals and execute them, and plans eventually bring about their effects in the world, possibly obstructing the goals of the agents or affecting their values. Values, in particular, are especially important for drama (Axiom 13) because they are the key to the appraisal of moral emotions (Battaglino et al., 2013; Lombardo et al., 2015b).

Notice that, in accordance with Statement C2: "Conflict can be among different agents or within the same agent. Therefore conflict can be interior or exterior to an agent.", Drammar does not pose any constraints on the agents who have the goals (and consequently intend the plans to achieve them), or on the agents whose value are engaged, so a conflict may concern different agents or may occur within the same agent (see the "Same or different" link at the top of Fig. 3, in red).

Figure 3 illustrates how conflicting plans (top of the figure) are linked to the values of the agents (Value class, on the right of the figure): the role of values in conflicts emerges because the agent's

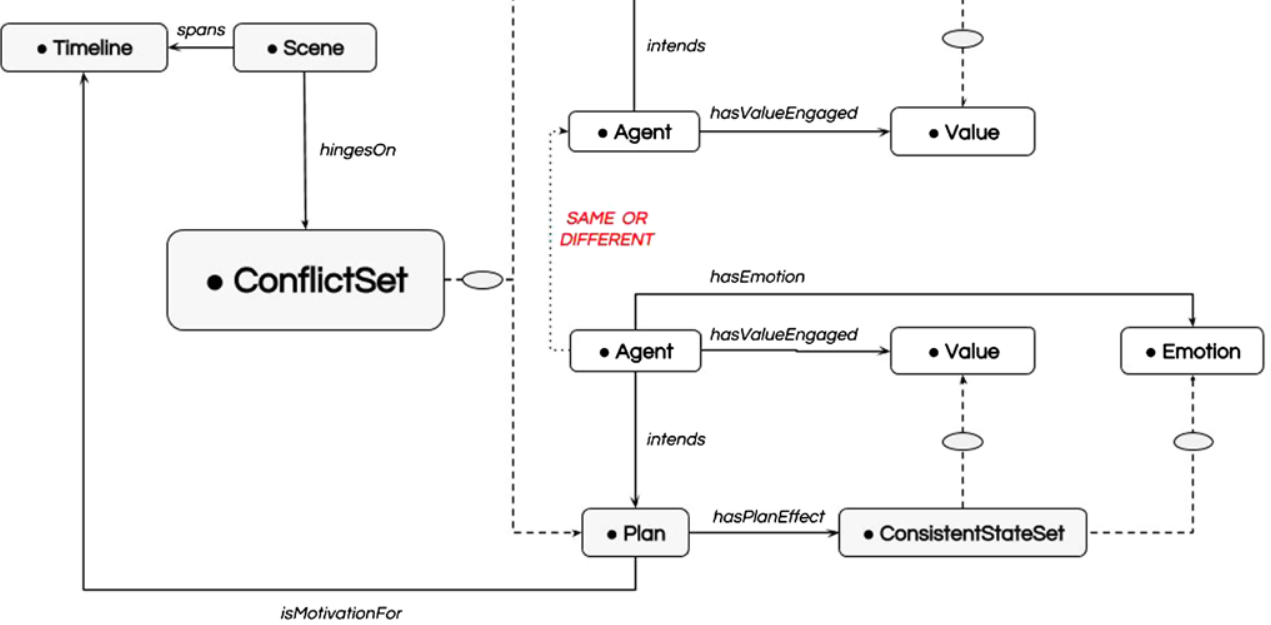

Fig. 3. The portion of the ontology concerning the notion of conflict. See Figs 7 and 8 in Appendix B for the symbols on the edges, representing the patterns for sets and lists. 
values are engaged by the effects of plans, meaning that their state is affected by the actions in the plan (see the hasValueEngaged property at the center of Fig. 3). This is in line also with Statement C3: "Conflict is linked to the nucleus of the agent's fundamental psychological traits", since in Drammar, psychological traits are intended as mental states, which include beliefs, goals, values and emotions.

In the literature about drama, conflicts are related with the emotional response of the characters, as stated in Statement C4, "Conflict provokes an emotional response in the agent". According to the model of emotion appraisal assumed by Drammar, the generation of emotions is determined by the relationship of the agent with the environment. This relation is modeled by the fact that emotions (represented by the Emotion class), a type of Mentalstate, can be part of the effects of plans and timelines, thus a direct relation between the agent's plans in conflict and their emotions can be established (see the right area of Fig. 3).

The contrast between the agents and the situation mentioned in Statement C5: "Conflict is the contrast between the agents and the situation" is modeled in Drammar through the relationship between the agent's plans and the timeline they motivate, where the effects of plans are transferred onto world states (the actual "situations" agents face). In order to enable this relationship, Drammar allows the same state to belong at the same time to the set of the effects (or preconditions) of a plan and to the set of the effects (or preconditions) of a timeline. ${ }^{12}$ The world state brought about by conflicting plans, then, is the "obstacle" mentioned in Statement C6: "Conflict is represented by an obstacle".

\subsubsection{Segmentation}

From the literature (Aristotle, 2013; Diderot, 1966; Freytag, 1863; Horace, 1989; McKee, 1997; Pfister, 1991; Polti, 1895; Spencer, 2002) we have distilled the following statements:

(S1) Drama can be segmented into parts. The segmentation of drama can be concerned with:

1. the plot;

2. the practical organization of the live staged event.

The parts that compose the drama are usually called:

1. Scene and Act - in theatre;

2. Scene, Sequence, Act - in film and TV.

(S2) The parts of the drama are organized hierarchically.

(S3) Each part, in each level, has the form of the whole drama (fractal recursion).

As illustrated in Section 2, drama orchestrates characters' intentional actions into well-specified shapes, acknowledged by the literature about drama. In particular, Statements S1: "The drama can be segmented in parts" and S2: "The parts of the drama are organized hierarchically" summarize the basic tenets about drama structure shared by most accounts. The modelling of drama structure, in Drammar, relies on three main notions, defined on top of the data structures included in the ontology (see Section 5.1):

- the Unit gathers a set of actions into a discrete segment, thus providing the basic element of drama organization. Units, the elementary components of drama structure, are represented as sets of actions (conveyed through some specific medium and language); formally, the Unit is a type of Set whose elements consist of actions.

\footnotetext{
${ }^{12}$ Notice that, although Drammar assumes the mapping of plans preconditions and effects onto timelines, it does not pose any constraints about the mapping between the two: the task of mapping the preconditions and effects of plans onto the preconditions and effects of timelines is left to a separate set of reasoning rules, as described in (Lombardo et al., 2015b).
} 


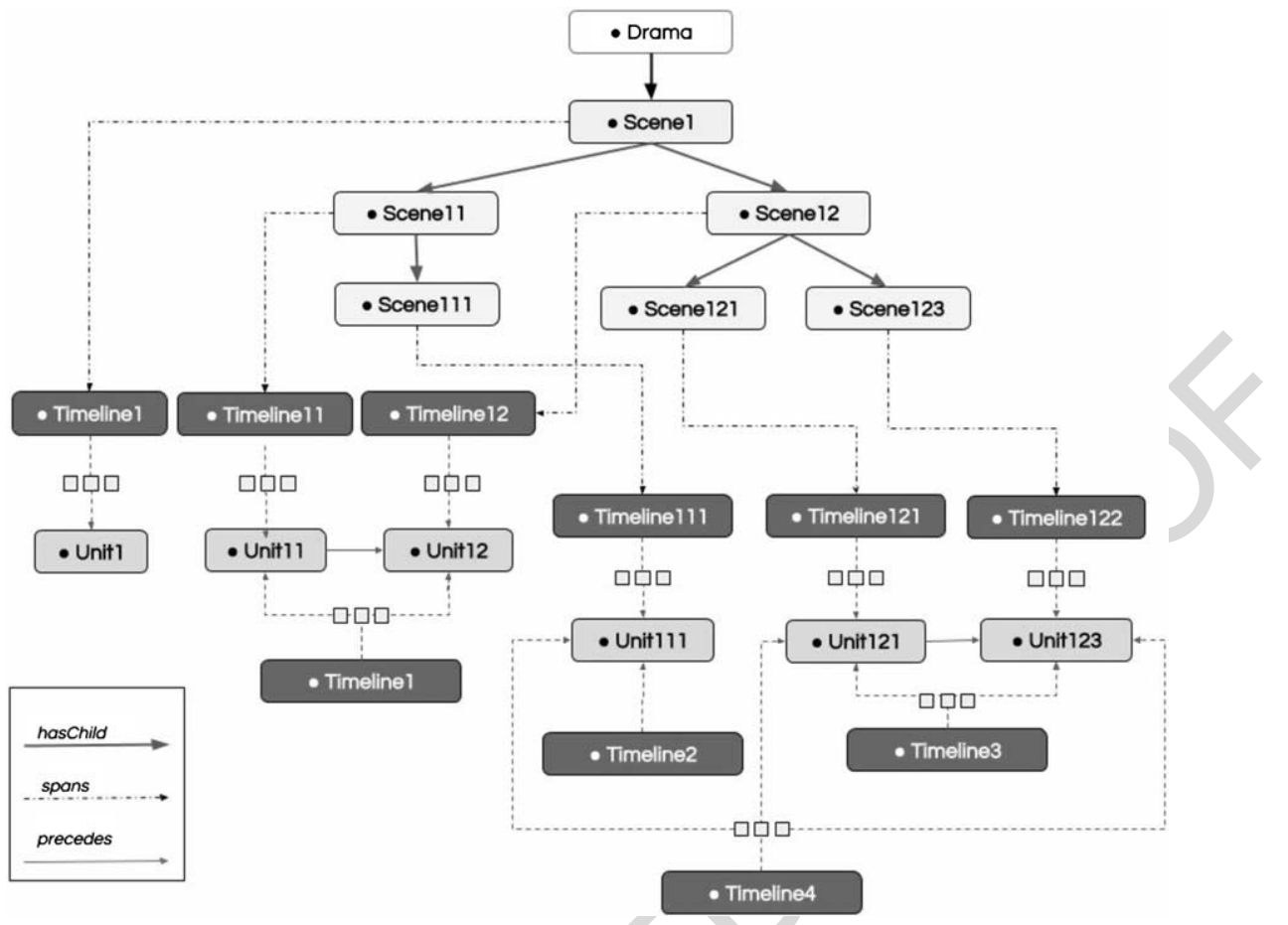

Fig. 4. The representation of the structure of drama in Drammar. The upper part of the figure illustrates the tree-shaped structure of the scenes in drama (related by hasChild properties); scenes span timelines, which organize action units in sequences where the units are related to each other by the precedes property (see Fig. 7 in Appendix B for the symbols on the edges, representing the pattern for lists). For the sake of readability, in this figure shades of gray have been used to represent the different structural elements: Dark gray for timelines, medium gray for units, light gray for scenes.

- the Timeline puts units into sequences to form larger scale operators that make the world advance through the changes brought about by the actions in them; units and timelines, together, form a pattern (illustrated in Fig. 1) that organizes actions into a sequence of discrete segments, according to Statement S1. The list data type is employed to superimpose sequences onto the segmentation of drama into units.

- the Scene corresponds to a given extension of the perceivable "text" of the drama in some medium. A scene can include smaller scenes, thus resulting in a hierarchy of scenes, as prescribed by Statement S2. The organization of drama into scenes is represented by the tree data type structure. The whole drama is an instance of the Tree class; the scenes are the nodes of the tree, and are instances of the TreeNode class. The has Child property connects every scene with its children scenes, until the leaves of the hierarchy.

The two structural aspects of the organization of drama, scenes and units, are not directly linked to each other, since they pertain to different aspects of the organization of drama: units pertain to the mimetic language of drama, made of actions; scenes pertain to the 'text' by which drama is exposed to the audience, and its organization. Scenes are mapped onto units through the Timeline class, which provides the interface between the two levels. Formally, a Scene spans a Timeline i.e., it corresponds to a given timeline, which in turn encompasses a sequence of units (Axiom 14). A specific type of scene, called DrammarScene, represents the notion of scene as the product of the agents' conflicts occurring 
along the timeline. The DrammarScene class, a subclass of the Scene class, is characterized by the fact that it hinges on a Conflictset, namely a set of conflicting plans (Axiom 15).

Finally, notice that, as prescribed by Statament S3: "Each part, in each level, has the form of the whole drama (fractal recursion)", the structure described above is repeated at each level of the scene hierarchy: scenes (nodes of the drama tree) span timelines, and they contain further scenes that in turn span other timelines, until the leaves of the tree are reached.

\subsection{Implementation}

The ontology is encoded in OWL2 (Motik et al., 2009b), following the Rule Language profile (OWL2 RL (Motik et al., 2009a)), due to its trade off between expressiveness and effectiveness of reasoning. OWL2 RL allows verifying ontology consistency, class expression satisfiability, class expression subsumption, instance checking, and conjunctive query answering problems in polynomial time with respect to the size of the ontology. In addition to this rationale, notice that the refinement of informal statements distilled from the literature about drama into the ontology language almost naturally leads to a formalization style where knowledge can be expressed as a system of rules, as assumed by this profile.

The ontology has a fair level of expressivity, ALCHIQ(D), namely ALC (no transitivity) with property hierarchy $\mathrm{H}$, inverse properties I, qualified cardinality restrictions $\mathrm{Q}$, and datatypes D.

The ontology consists of 51 classes, 66 object properties and 18 data properties. A large majority of classes (32 out of 51) are defined as equivalent classes, in the attempt to accurately describe the nature of the core elements of drama and verify the ontology through classification tasks.

\section{The encoding of a drama}

In the previous section, we described how the knowledge about drama has been encoded into Drammar, leading to a formal conceptualization intended as a set of core elements, their properties, and the relations over them. In this section, we illustrate the use of Drammar for the encoding of a dramatic work. To do so, we resort to a specific scene, the well known "nunnery scene" of Shakespeare's Hamlet, (Third Act, First Scene). ${ }^{13}$ At the beginning of this scene, Ophelia tries (twice, lines 95 and 100) to give back the gifts received from Hamlet in the past, and Hamlet negates to have given such gifts (lines 95-96):

OPHELIA

93 My lord, I have remembrances of yours,

94 That I have longed long to re-deliver; 95 I pray you, now receive them. HAMLET No, not I; ${ }^{14}$

96 I never gave you aught.

OPHELIA

97 My honor'd lord, you know right well you did;

98 And, with them, words of so sweet breath composed

99 As made the things more rich. Their perfume lost,

100 Take these again; for to the noble mind

${ }^{13} \mathrm{http}: / /$ shakespeare-navigators.com/hamlet/H31.html

${ }^{14}$ Line 95 is split between Ophelia's turn "I pray you, now receive them" and Hamlet's abrupt reply "No, not ...". 
101 Rich gifts wax poor when givers prove unkind.

102 There, my lord.

In this scene, Ophelia is sent to Hamlet by Polonius (her father) and Claudius (Hamlet's uncle, the king) to confirm the assumption that Hamlet's madness is caused by his rejected love. According to the two conspirators, Ophelia should induce him to talk about his feelings. At the same time, Hamlet tries to convince Ophelia that the court is corrupted and that she should go to a nunnery. In the climactic part of the scene, Hamlet puts Ophelia to a test to verify her honesty: guessing (correctly) that the two conspirators are hidden behind the curtain, he asks the girl to reveal where her father Polonius is. She decides to lie, by replying that he is at home. Hamlet realizes from the answer that also Ophelia is corrupted and consequently becomes very angry because he now thinks that there is no hope to redeem her. The encoding described here is situated at the core of the scene, where Hamlet tests Ophelia's honesty by asking rhetorically "Where is your father?" (Hamlet knows that Polonius is in the same room where they are, behind a curtain), and Ophelia lies by replying "At home, my lord" (see also Damiano and Pizzo (2008) for a detailed description of this interpretation).

The encoding of a dramatic work consists in the identification within the work of the individual entities formalized by the ontology Drammar and of their consequent instantiation; this encoding proceeds from an interpretation process that analyzes the text and lists the incidents that occur in it. Lombardo et al. (2017b) argued that Drammar can encode the abstraction of drama into a digital item, and showed how the obtained representation can support different conceptualizations of drama, being theory-neutral, oriented to the preservation of drama as intangible cultural heritage.

Figure 5 provides an overview of the structure of the nunnery scene (the RDF graph of the example can be found in Appendix C). For space reasons, only the most relevant individuals and relations are represented in the figure. The figure is divided into four regions, which correspond, respectively, to the four main elements of drama:

- the structure of drama (top area), namely scenes and timelines;

- the characters' conflicts (left area), carefully orchestrated from the agents' plans;

- the actions (right area) performed by the characters;

- the characters, or agents (bottom right area).

Structure. The top area of the figure (labeled as "structure") shows the articulation of the nunnery scene into sub-scenes, and the organization of actions into timelines. In particular Fig. 5 represents the scene ( $S_{-}$WhereQuestion), which hinges on the conflict between Ophelia's plan to save his father's authority and Hamlet's plan to learn about Ophelia's honesty, that he accomplishes through a test "Where is your father" to which she answers with a lie "At home, my Lord". Scenes span timelines of incidents: here, scene $S_{-}$WhereQuestion spans timeline TL_Units1011, which contains shorter timelines (TL_Unit10 and $T L_{-}$Unit11), each encompassing a single unit (the one where the character's action is executed). The last timeline in the scene, $T L_{-}$Unit11, puts at stake Hamlet's value of honesty, as represented by its effects (CSS_Eff_TL_unit11).

Conflicts. The left area of the figure (labeled as "conflicts") represents the conflicts over the agents' plans, namely the conflict (CS_WhereQuestion) between Hamlet's plan of learning about Ophelia's honesty $\left(P_{-} H_{-}\right.$LearningHonesty) and Ophelia's plan to respect her father's authority $\left(P_{-} O_{-}\right.$Saving $P$ Polonius_Authority), which are incompatible. Each plan subsumes a simpler, directly executable plan $\left(P \_H_{-}\right.$ AskR for Hamlet and $P \_O \_L i e$ for Ophelia), which contains the action that the character performs in the scene. 


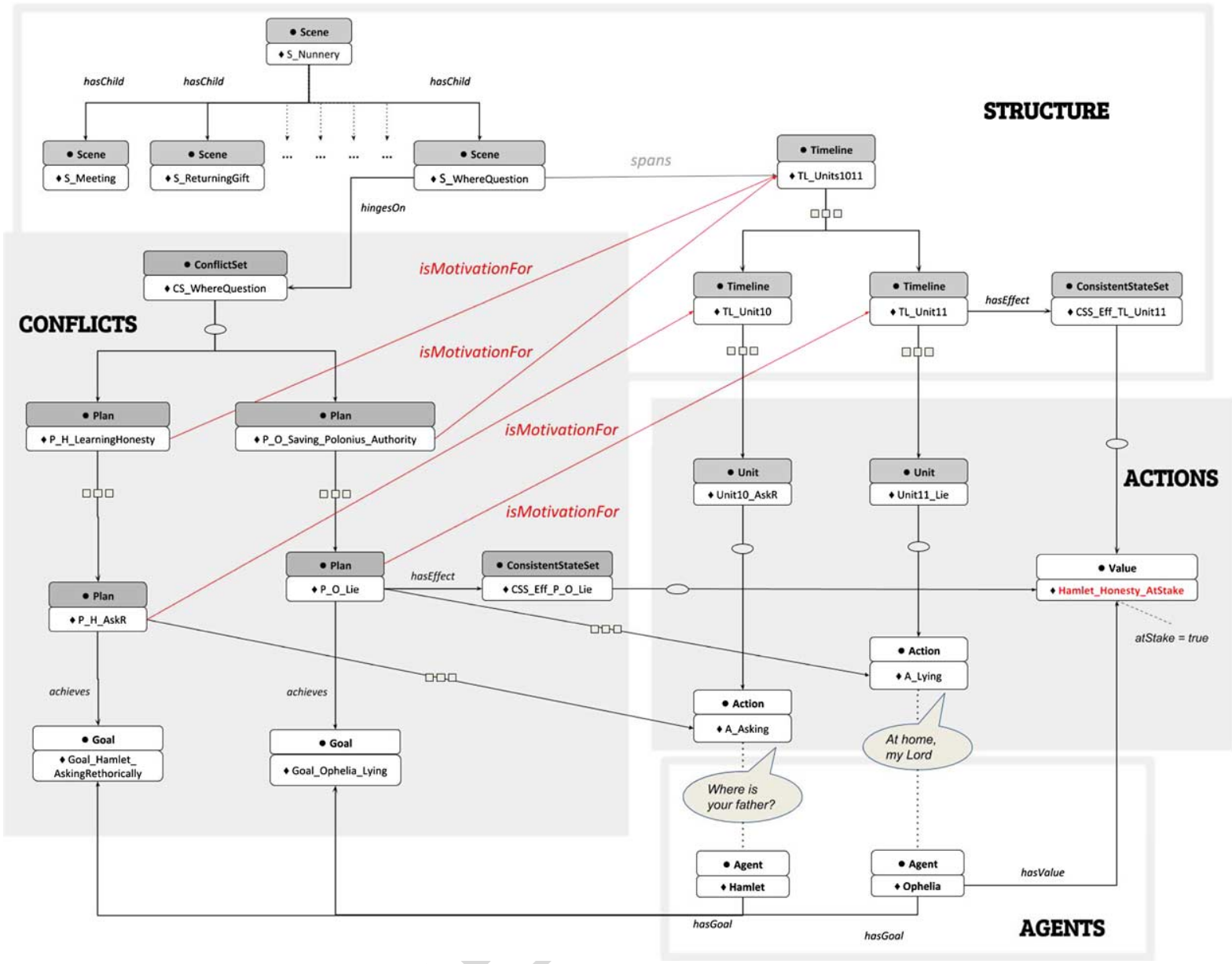

Fig. 5. The dramatic structure of the example scene. The boxes outline the patterns for the four core elements of drama: Structure, conflict, action and agent. For the sake of readability, the patterns for sets and lists have been represented in a simplified way according to the conventions illustrated in Appendix B.

Actions. The right part of the figure ("actions") shows the actional units, each containing one or more agents' actions. Unit TL_Unit10 contains Hamlet's asking action (A_Asking); unit TL_Unit11 contains Ophelia's lying action (A_lying). The enactment of actions by the agents, formally encoded by the role structure described in Section 5.2, is represented here by the dashed lines connecting the actions with the agents at the bottom of the figure for space reasons.

Agents. Besides performing actions, agents (Hamlet and Ophelia) have goals and values. The former are connected to plans, as represented by the hasGoal properties that connect high level plans with agents; the latter (hasValue properties) can be put at stake by the agents' plans, once their effects are actualized in a timeline.

Notice that the meaning of the scene stems not only by the encoding of the single elements, but from the relations over them, represented in Fig. 5 by the properties that connect the different areas:

- The scene hinges on the conflict between Ophelia's plan to save his father's authority and Hamlet's plan to learn about Ophelia's honesty; this is represented by the hingesOn property that con- 
nects scene $S \_$WhereQuestion to element $C S \_$WhereQuestion (instance of class Conflict Set in Drammar);

- Plans (in conflict) motivate the actions which, gathered in units, form the timelines: longer timelines are motivated by higher-level plans, while shorter timelines are motivated by lower-level plans, so that each sequence of actions in drama is assigned its meaning in terms of intentions (see the tilted edges labelled as isMotivationFor connecting the plans on the left of the figure with the timelines on the right of the figure).

- Actions play a two-fold role: planned by the agents in their plans, they are executed by them in the units. This is encoded by having the same actions contained in both plans and timelines (through units): Ophelia's lying action is not only part of her lying plan, but is also contained in a unit (unit 11), whose effects, through the timeline it belongs to, put Hamlet's value of honesty at stake. Notice that putting Hamlet's value at stake is an effect of the plan, but it does not generate any effects if not through the timeline that it motivates.

\section{Using Drammar}

As anticipated in the Introduction, the development of Drammar has been carried out for a decade by a team of knowledge engineers, experts in Artificial Intelligence, and drama scholars, by interleaving use and design as a consequence of the insight gained through practical tasks. As a resource, Drammar was employed for various tasks, ranging from the visualization of drama structure for research and teaching (Lombardo and Pizzo, 2016; Albert et al., 2016) to the calculation of characters' emotions (Lombardo et al., 2015b).

Each of the tasks mentioned above relies on a specific workflow, achieved through the participation of different roles (annotators, drama experts, AI scholars, communicators, etc.), and involves the creation of documents where the content of dramatic works is annotated in Drammar terms. Since the encoding of dramatic content in ontology languages is time-consuming and cannot be carried out by those who don't have a training in semantic technologies, in order to alleviate this task and make it accessible to drama scholars and practitioners, we launched the POP-ODE initiative (POPulating Ontology Drama Encodings), described by Lombardo et al. (2017a)). Developed in an iterative fashion through the interaction with the users (graduate students, teachers and scholars from the School of Art and Media at the University of Turin), POP-ODE consists of a pipeline and a number of tools for the annotation of content metadata of dramatic works. The annotator works through a web-based interface, filling the tables of a database built according to the tenets of ontology Drammar. A mapper module, called DB2OWL, converts the database tables into an OWL file, termed Drammar Instantiated Ontology (DIO) file. A software module, called OWL2CHART, extracts the individuals and their properties into a XML file, which is then converted in an interactive visualization by the interactive chart module. The interactive visualization allows the exploration of the actional structure of a dramatic work with respect to its segmentation, showing how the characters' intentions motivate each segment of the drama. For example, in our running example (the "Nunnery scene" from Shakespeare's Hamlet, see Section 6), Ophelia's plans span longer subsequences of the timeline, and their beginning is always followed by the beginning of Hamlet's plans, signaling the fact that he is mostly reactive in this scene, while she has the initiative for most of the time.

The annotator is only required to informally know the tenets of the model underlying the annotation, which are reflected in the annotation interface. In order to enable a crowdsourcing-based schema in the management of the annotation projects, POP-ODE has been developed and deployed as an online system. 
The feasibility of the annotation has been tested through the creation of a corpus of annotated dramatic media, spanning from movie scenes and marketing videos to video clips and scripts (see Lombardo et al. (2017a) for details). Albert et al. (2016) have exploited the annotation of a 19th Century dramatic work in Drammar (Nikolay Pogodin's "Kremlin Chimes") to prove the expressiveness of the ontology for the methodology of action analysis proposed by the drama theorist Maria Knebel (Carnicke, 2010) and to test its use from the perspective of the actors' interpretation.

The status of the annotated works created by the scholarly and production activities mentioned above can be clarified by referring to the model known as Functional Representation of Bibliographic Records, or FRBR (O'Neill, 2011). Devised for the bibliographic domain, FRBR has been exploited also in artistic domains, such as music and theatre, to account for the complex relations that connect the work of the author with its interpretation by performers. In particular, according to Doty (2013), an account of these relations is necessary to guarantee the recognisability of the performance with respect to the production it belongs to, i.e., to acknowledge the specific features of each of multiple productions of the same work while keeping track of their relations with the original work. The FRBR model acknowledges four main entities: Work, i.e., abstract ideation, Expression, i.e., the encoding of the Work in a specific language, Manifestation, i.e., the embodiment of the Expression in a concrete representation, and Item, a single instance of the Manifestation. For example, with reference to Beethoven's Ninth Symphony, Beethoven's idea of the Ninth Symphony is a Work; the interpretation of the Ninth Symphony by the Berliner Philarmoniker is an Expression; a recording of the Ninth Symphony by the Berliner Philarmoniker is a Manifestation; and the single recording of the Berliner Philarmoniker's performance in a medium (e.g., a CD) is an Item. Notice that, in the framework of FRBR, the interpretation of the Ninth Symphony by the Berliner Philarmoniker proceeds from Beethoven's expression of his work in the musical language (manifested through its encoding in a score), keeping the entities generated by the interpretation process (e.g. the recordings) separated from the entities generated by the creative process accomplished by the author (e.g., the scores).

In a similar way, the representation of drama provided by Drammar is conceptually situated at the level of Expression in FRBR: issued from an interpretation activity of the original work conceived of by the author, it consists of an abstract linguistic entity encoded in OWL, that can be subsequently serialized in a specific format among those encompassed in the specification of OWL, and finally transferred into a digital resource. Figure 6 shows the role played by the annotation in the FRBR model through the example of Shakespeare's Hamlet: both Hamlet as Shakespeare's theatrical screenplay and Laurence Olivier's movie (Hamlet, 1948) can be described as Works that have their own Expressions, expressed respectively in iambic English and as a film. The dataset described using the vocabulary provided by Drammar, then, is the Expression of Hamlet as an intangible work, which ideally approximates the expression of Shakespeare's Hamlet, develops into its own Manifestation, and can be distributed as a set of Items, thus effectively contributing to the preservation of the original work as proposed by Lombardo et al. (2016). In terms of FRBR (Fig. 6, left), the representation of a dramatic work in Drammar results in a derivative Work that abstracts from the existing work the elements acknowledged by Drammar (agent, action, conflict and structure); such a Work is expressed through an instantiated ontology document, the Drammar Instantiated Object (DIO, top left), and has a Manifestation in a specific encoding of the ontology - for example, an RDF/XML file published on the Web - which can be visualized as a single Item on a specific browser by a given person (bottom left).

Resources of this kind are claimed to be of great importance for the researchers in the digital humanities: as discussed by Varela (2016), the notion of theatre and drama does not manifest in an item 


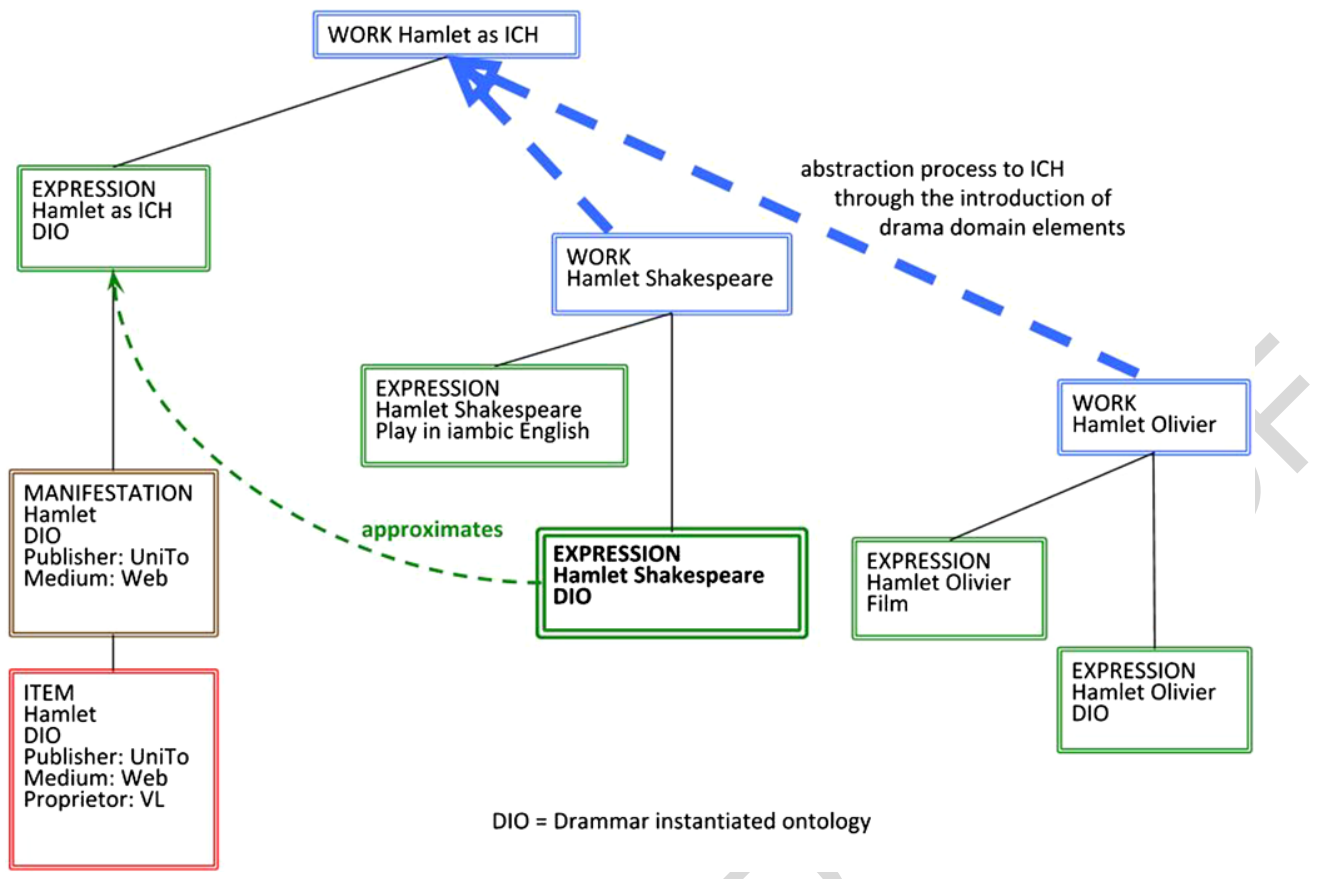

Fig. 6. The annotation of drama within the conceptual framework of FRBR.

or an event sufficiently unified and standardized to be represented via a conventional database. Semantic web technologies, and ontologies in particular, are suitable to represent performance interpretations through the possibility of sharing the terminology through several approaches and the possibility for instances to belong to multiple classes scattered through several ontologies, though maintaining the original meaning cross-culturally. Semantic web technologies and ontologies in particular are suitable to represent disagreement in performance interpretations, since their fractal nature addresses the atmosphere of "sophisticated disagreement" that characterizes performance research (Varela, 2016, p. 136), allowing several aspects of these disagreements to be captured "in a structured, systematic way" (Varela, 2016, p. 139).

\section{Related work}

Several research lines are relevant to the formalization of drama, ranging from cultural heritage dissemination to the indexing and search of media repositories. In particular, here we will focus on three main fields of research: computational models of story and narrative, including story ontologies; semantic annotation of multimedia; and semantic initiatives for cultural heritage.

Story ontologies have been proposed with two main goals, namely the purpose of classifying story types and the purpose of providing an underlying model for narrative annotation. A well known example of the first type of systems is the work of Gervás et al. (2005) (see also Gervás (2013)). In this work, inspired by the structuralist account of the tale provided by Propp (1968), an OWL ontology of fairy tales is exploited to model different plot types. The system uses the ontology to perform case-based reasoning and generate new tales. In the same line, the work of Hartmann et al. (2005) uses automatic classification 
techniques to classify plot types; the Opiate system (Fairclough, 2004) relies on a Proppian model of story to create and populate story worlds. A formalization of Propp's model is also described by Gervás (2013): in this work, a computational system exploits the formal model to generate new stories in the style of Russian fairy tales. Differently from previous attempts to formalize Propp's theory, this proposal constitutes a more rigorous description of the original model in computational terms. However, the use of Propp's theory as a general story model has been questioned by several authors, especially in relation with the new media (Cavazza and Pizzi, 2006; Tomaszewski and Binsted, 2007).

Overcoming the differences across media types and genres is one of the main challenges faced by the research on media annotation. In this field, story ontologies have been proposed as a way to provide a shared and inter-operable model for annotation scenarios which rely on the paradigm of crowd-sourcing and are characterized by the presence of different types of narrative contents. The OntoMedia ontology provides a media-independent model, designed to annotate the content of different media objects in terms of characters, events, locations, etc., ranging from written literature to comics and TV fiction (Jewell et al., 2005b; Lawrence, 2011). The OntoMedia ontology contains a very detailed model of the story, tailored on story annotation, and mainly focused on the representation of the events and the order in which they are exposed. Developed in collaboration with the BBC for the application in fields as news, drama, and historical facts, is employed to annotate plot elements across the episode storylines of the Dr. Who sci-fi TV series. OntoMedia lends itself to the comparison of cross-media versions of the same story (for example, a novel and its filmic adaptation); it is an event- (instead of character-) based description of the timeline of story incidents, with no interpretive intents, and so it does not cover the description of characters in terms of goals and intentions. In a complementary way, the Story Intention Graph proposed by Elson (2012) relies on the representation of the short-term characters' intentions to build an interpretive layer of a narrative text, but it does not account for the causal sequence of the drama in terms of long-term intentions.

In recent years, research on story formalization has put forth formal accounts of narratives and drama that do not rely on ontology languages. In particular, Szilas (2016) describes a language of symbols and relations that can be employed to express the "paradoxical structures" underlying simple stories. This model has been applied to a corpus of Aesop's fables, and the annotation has been employed to measure the interestingness of stories as a function of their structural elements (Szilas et al., 2016). Drawing from the anthropological tradition of folklore studies and by the empirical directives issued by scriptwriting theories, the vocabulary underlying this model combines an intention-based account of story with elements such as parental relations, tasks and obstacles. Targeted at an abstract conceptualization of the story, this model is not tailored to the annotation task; with respect to Drammar, it is situated at a higher level of abstraction, with primitives that may be partly derived from the interplay of characters' intentions expressed in Drammar as conflicts. Mainly geared to story generation, the Impulse system proposed by Eger et al. (2015) relies on first-order logic to describe the content of stories, focusing on the temporal representation of the actions and events. By encoding the notion of characters' mental spaces, this system is able to derive and maintain, through reasoning techniques, the mental state of the single characters in terms of beliefs and intentions. In this sense, its aim partly overlaps with Drammar; however, its current expression in a logical format would require some adaptation to integrate it in the open Web of Linked Data.

The semantic annotation of media is one of the main applications of ontologies, aimed a reducing the semantic gap between the data about signal they contain and the high-level content encoded in these data. Since drama is often conveyed through media (from films and performance recordings to radio drama), representing the multimedia data is a necessary step to cope with the semantic gap in the perspective of 
subsequent applications. Vocabularies of semantic descriptors represent the most straightforward way to annotate media; they include multimedia vocabularies, such as TRECVID (Naphade et al., 2006), and more sophisticated tools, such as the VERL ontology (François et al., 2005). Semantic representations of media content usually rely on low level representations of multimedia data (typically, video) for indexing purposes, with the goal of making the annotated media searchable by access tools. However, these representations usually address the occurrence of basic events in the audiovisual stream and/or the appearance of physical entities, such as a person who is running, or a falling object; situated at a lower level of abstraction than Drammar, they do not address the narrative or dramatic function of the annotated events. With respect to this approach, Drammar is situated at a meaning level (i.e., dramatic structures) that is conceptually more abstract. As shown in Section 6, however, semantic descriptors of entities and events can be coupled with the description of incidents provided by Drammar, with the goal of anchoring the annotation of drama into multimedia data, thus reducing the semantic gap from the signal level to its ultimate meaning for the audience.

Considering drama as a type of cultural heritage, the Conceptual Reference Model of cultural heritage, issued by the CIDOC working group of the International Council of Museums (ICOM), and known as CIDOC CRM (Doerr, 2003; Doerr and LeBoeuf, 2007), is relevant. CIDOC CRM is intended as a "common language for domain experts and implementers", specifically aimed at the design of information systems in the field of cultural heritage (Doerr, 2003). CIDOC CRM describes cultural heritage not only as a set of artifacts, but also in terms of the real world entities that are relevant to the art domain, and to the functioning of museums in particular. In the terms of CIDOC CRM, the digital objects describing a drama item, encoded in Drammar, can be seen as instances of the Information Object class. The latter is a subclass of both Propositional Object and Symbolic Object classes, and may include an instance of a movie or as well as a set of equations, and even accommodate graph-like structures (such as, e.g., ontology-based representations). The physical counterpart of the abstract encoding, then, would be described as an instance of Physical Man Made Thing, i.e. physical items generated by human activity. Situated at a different conceptual level than Drammar, CIDOC CRM provides a representation of cultural heritage objects that is functional to the management and planning of the processes that are relevant to their acquisition, maintenance, preservation, and curation, and does not deal with the representation of the content of cultural heritage objects.

\section{Conclusion}

In this paper, we presented an ontology of drama called Drammar. Drammar has been designed to systematize in a formal language the conceptual model of the entities that essentially form a drama and underpin the extremely various manifestations of drama observable through the centuries across different media and genres. Drammar aims at providing an authoritative knowledge source about drama, distilled from an extensive survey of the literature carried. Developed with an eye on the annotation of dramatic works, the ontology avails itself of the tools and methodologies provided by the Semantic Web project over more than a decade, which range from formal languages for encoding ontologies to ontology engineering methodologies.

Drammar is part of a larger interdisciplinary research initiative, brought about for more than a decade, aimed at collecting data about drama through the annotation of a corpus of dramatic works. With respect to this initiative, Drammar provides the core of a crowdsourcing workflow developed for alleviating the task of annotating drama by experts who do not have a background in formal ontologies (Lombardo et al., 2017b). The ultimate goal of annotation is twofold: on the one side, it is aimed at collecting ground truth 
data about drama; on the other side, it provides the way to verify against data the knowledge encoded in the ontology, thus possibly evolving the ontology itself towards more effective and accurate accounts of drama, informed on its manifestations.

The evolution of Drammar is open to the contributions of an interdisciplinary community which ranges from drama experts and theorists to developers of natural language resources for the study and analysis of media. We expect that the ontology will be subject to the addition of specialized modules as an effect of its exploitation by different communities on different tasks, and that its relationships with linguistic resources will be strengthened through its use in more annotation initiatives. The current version of Drammar is available at the URI http://www.purl.org/drammar. More details about the theoretical foundations of the drama domain and how they were incorporated into the design of the ontology can be found at http://www.cirma.unito.it/portfolio_page/drammar/.

\section{Acknowledgements}

We thank our colleagues for their contributions to Drammar along years, from theoretical discussion to help with data. In particular, we thank Dr. Antonio Lieto (University of Turin), Dr. Giacomo Albert (University of Turin), Dr. Cristina Battaglino (Nuance Co.), Dr. Carmi Terzulli. Also, we thank Davide Pulizzotto (University of Montreal, CA) for the contributions he provided as a visiting scholar at the Department of Computer Science in Turin. The current form of the ontology is the result of the endless exchange of ideas with all of them, and of the insights they provided. Last, but not least, we are in debt with the reviewers who contributed in a significant way to give the paper its final shape with their helpful insight and valuable advice.

\section{Appendix A. List of axioms}

We adopt the Manchester syntax (https://www.w3.org/TR/owl2-manchester-syntax/) for encoding the axioms, since it is universally considered more user-friendly than other serializations. Also, we use the following conventions for improving the readability of the axioms: OWL language elements are in bold, classes are typewriter font, properties are in italic.

(1) Action equivalentTo Process and (isMemberOf some Unit) - an action is a type of process that belongs to a unit (remember that the unit is type of Set, see below);

(2) Unit equivalentTo Set and (isOrderedBy some Timel ine) and (hasMember some (Action and (isDescribedBy some (FrameNet Schema and (hasRole min 1 (Role and (hasFiller some Agent)))))) - this axiom requires a Unit to be contained in at least one timeline, and to contain at least one action enacted by an agent: namely, an action whose description (encoded through a FrameNetSchema includes at least one role (property hasRole) having an agent as filler hasFiller).

(3) Timeline equivalentTo List and (hasTimelineEffect some ConsistentStateSet) and (hasTimelinePrecondition some ConsistentStateSet) and (containsOLE only (Ordered ListElement and (hasData some Unit)) - a Timeline is a sequence of Units and has preconditions and effects.

(4) Plan equivalentTo List and (isIntendedBy some Agent) - a plan is a list (of actions) intended by some agent; notice that the relation of the Plan class with the Agent class is inherited by its subclasses, namely AbstractPlan and DirectlyExecutablePlan; since this class subsumes different plan types, including underspecified plans, its definition does not require the containment of actions.

(5) AbstractPlan equivalentTo Plan and (containsOLE some (OrderedListElement and (hasData some $\mathrm{Plan})$ )) and (isMotivationFor some Timeline) - an abstract plan is a plan that i) achieves a goal, ii) is given by a sequence (or list) of plans, iii) motivates a timeline;

(6) DirectlyExecutablePlan equivalentTo Plan and (containsOLE some (OrderedList Element and (hasData some Action))) and (isMotivationFor some Timeline) - a directly executable plan is plan that i) achieves a goal, ii) consists of a sequence (or list) of actions, iii) motivates a timeline. 
(7) Agent equivalentTo DramaEndurant and (intends some Plan) - the agent is defined as a type of Endurant which is committed to (property intends) a plan.

(8) Role equivalentTo DescriptionTemplate and (isRoleOf exactly 1 FrameNetSchema)) and (hasFiller some (DramaEndurant or DramaPerdurant)) - each Role belongs to exactly one description schema of type FrameNetSchema and is filled by a DramaPerdurant (namely, an agent or object).

(9) Emotion equivalentTo MentalState and (isDescribedBy exactly 1 EmotionSchema) - emotions are a type of mental states and are described by a specific type of SituationSchema.

(10) State equivalentTo DramaPerdurant and (isDataOf some (SetMember and (isMemberOf some Consistent StateSet))) - this axiom expresses the constraint, inherited by the Mentalstate class, that a state can only exist within a consistent state set (i.e., in preconditions or effects of a plan or timeline). Emotions, as a type of mental states, can occur as well only within the effects of plans and timelines.

(11) ConsistentStateSet equivalentTo Set and ((isPlanEffectOf exactly 1 Plan) or (isPlanPreconditionOf exactly $1 \mathrm{Plan}$ ) or (isTimelineEffect Of exactly $1 \mathrm{Timel}$ ine) or (isTimelinePreconditionOf exactly $1 \mathrm{Timel}$ ine)) and (hasMember some (SetMember and (hasData some State)) - sets of consistent states (instances of the class ConsistentstateSet) contain only states and form the precondition or effects of timelines and plans (and can only exist in relation to them).

(12) ConflictSet equivalentTo Set and (hasMember some Plan) - a conflict set is a set of plans;

(13) Value equivalentTo MentalState and ((atStake value false) or (atStake value true)) and (isDataOf some (SetMember and (isMemberOf some ConsistentStateSet))) - a character's value (Value) is at stake or is in balance within the effects (or preconditions) of a plan or timeline.

(14) Scene equivalentTo TreeNode and (spans some Timeline) and (haschild only Scene) - a scene is a node of the drama tree (namely, an instance of the TreeNode class) which may contain other scenes as its children nodes (has Child property), and it corresponds to the extent (spans property) of some timeline.

(15) DrammarScene equivalentTo Scene and (hingesOn some Conflict Set) - a Drammar scene hinges on a conflict set (a set of conflicting plans).

\section{Appendix B. Graphic conventions}

Fig. 7. The List pattern (left column), its use in Drammar for representing plans and timelines (center) and their simplifications (right column). The arrow with the list symbol replaces the connection between the list and its items, realized through the OrderedListElement class in Drammar (omitted). 

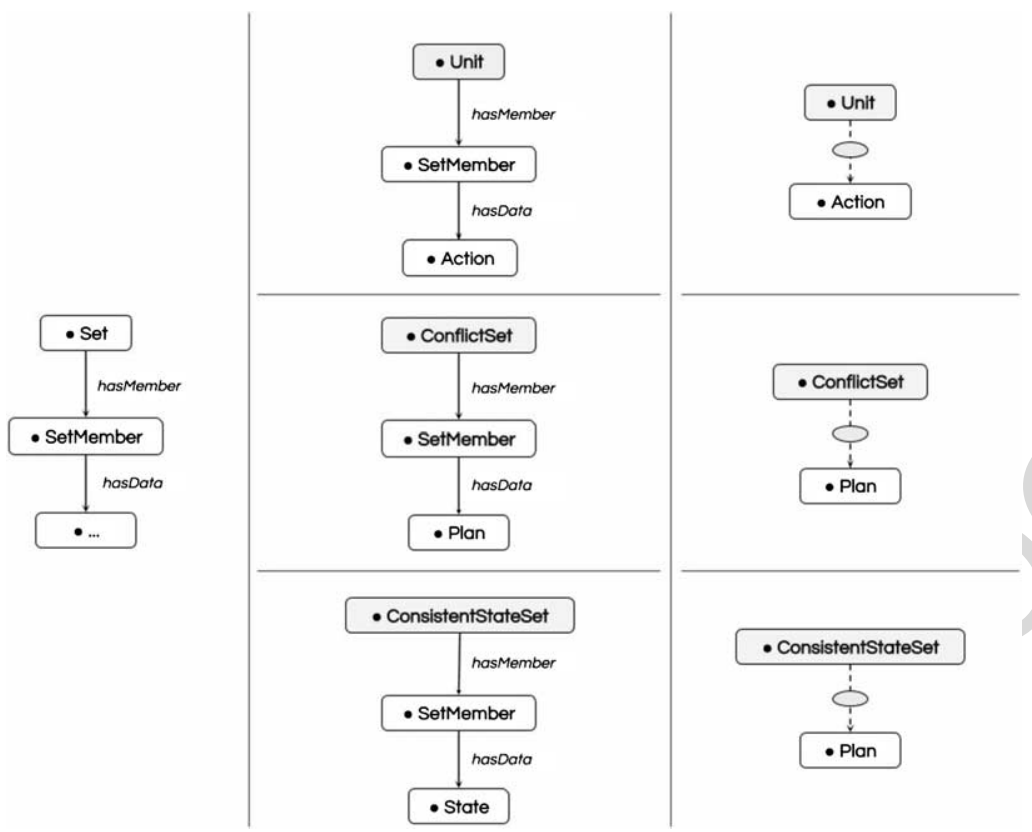

Fig. 8. The Set pattern (left column), its use in Drammar for representing units, conflicts and state sets (center) and their respective simplifications (right column). The arrow with the set symbol replaces the connection between the set and its members, realized through the OrderedListElement class in Drammar (omitted).

\section{Appendix C. Encoding of the example}

In this appendix, we report the portions of the annotation graph represented in Fig. 5 (for space reasons, set and list patterns have been omitted, following the same policy that has inspired the graphical abbreviations of Appendix B). All examples are represented in Turtle format (https://www.w3.org/TR/turtle/). ${ }^{15}$

\#\#\# http://www.purl.org/drammar\#S_whereQuestion

drammar:S_WhereQuestion raf:type owl:NamedIndividual, drammar: Scene ;

drammar:hingesOn drammar:CS_WhereQuestion ; drammar:spans drammar:TL_Units1011.

\section{\#\#\# http://www.purl.org/drammar\#CS_WhereQuestion}

drammar:CS_WhereQuestion rdf:type owl:NamedIndividual, drammar:Conflictset ;

rdfs:comment "Hamlet tests Ophelia's honesty by asking where is Polonius, and she fails the test by lying."^^xsd:string.

\#\#\# http://www.purl.org/drammar\#P_H_LearningHonesty

drammar:P_H_LearningHonesty rdf:type owl:NamedIndividual ,

${ }^{15}$ In order to enhance readability, the indentation of the triples has been slightly modified with respect to the formatting standards for space reasons. 
drammar:Plan ;

drammar:isMotivationFor drammar:TL_Units1011; rdfs:comment "Hamlet intends to test Ophelia for honesty" ^^xsd:string.

\#\#\# http://www.purl.org/drammar\#P_H_AskR

drammar:P_H_AskR rdf:type owl:NamedIndividual ,

drammar:Plan ;

drammar:inConflictWith drammar:P_O_Lie ;

drammar:isMotivationFor drammar:TL_Unit10 ;

drammar:accomplished "true"^^xsd:boolean ;

rdfs:comment "Hamlet intends to ask Ophelia about Polonius" location" $\wedge \wedge x$ s:string .

\#\#\# http://www.purl.org/drammar\#Goal_Hamlet_AskingRethorically

drammar:Goal_Hamlet_AskingRethorically rdf:type owl:NamedIndividual , drammar:Goal ;

drammar: isAchievedBy drammar:P_H_AskR ;

drammar:isDescribedBy drammar:GS_H_AskingRethorically ; rdfs:comment "H. wants to ask to O. about Polonius' Location".

\#\#\# http://www.purl.org/drammar\#P_o_Saving_Polonius_Authority

drammar:P_O_Saving_Polonius_Authority rdf:type owl:NamedIndividual , drammar:Plan

drammar:isMotivationFor drammar:TL_Units1011;

drammar:accomplished "true"^^xsd:boolean ;

rdfs:comment "Ophelia intends to respect her father's authority" $\wedge \wedge x$ sd:string

\section{\#\#\# http://www.purl.org/drammar\#P_O_Lie}

drammar:P_O_Lie rdf:type owl:NamedIndividual , drammar:Plan ;

drammar:containsOLE drammar:OLE_A_men_03_in_P_O_Lie ; drammar:hasPlaneffect drammar:CSS_Eff_P_O_Lie ;

drammar:isMotivationFor drammar:TL_Unit11 ;

drammar:accomplished "true"^^xsd:boolean ;

rdfs:comment "Ophelia intends to lie about Polonius' location"

$\wedge \wedge$ xsd:string.

\#\#\# http://www.purl.org/drammar\#CSS_Eff_P_O_Lie

drammar:CSS_Eff_P_O_Lie rdf:type owl:NamedIndividual , drammar: ConsistentStateset.

\#\#\# http://www.pur1.org/drammar\#Goal_Ophelia_Lying

drammar:Goal_Ophelia_Lying rdf:type owl:NamedIndividual , 
drammar:Goal ;

drammar:isAchievedBy drammar:P_o_Lie;

drammar:isDescribedBy drammar:GS_O_Lying ;

drammar:Goal_type "Perform"^^xsd:string ;

rdfs:comment "O. wants to lie about Polonius' location."^^xsd:string .

\#\#\# http://www.purl.org/drammar\#TL_Units1011

drammar:TL_Units1011 rdf:type owl:NamedIndividual , drammar:Timeline ;

drammar:containsFirstoLE drammar:OLE_TL_Units1011_Unit10 ; drammar:containsLastoLE drammar:OLE_TL_Units1011_Unit11 ;

\section{\#\#\# http://www.purl.org/drammar\#TL_Unit10}

drammar:TL_Unit10 rdf:type owl:NamedIndividual, drammar:Timeline ;

\section{\#\#\# http://www.purl.org/drammar\#Unit10_AskR}

drammar:Unit10_AskR rdf:type owl:NamedIndividual drammar:Unit ;

rdfs:comment "Hamlet tests Ophelia with the question about her father's location."^^xsd:string.

\#\#\# http://www.pur1.org/drammar\#A_Asking

drammar:A_Asking raf:type owl:NamedIndividual , drammar:Action ;

drammar:isDescribedBy drammar:PS_questioning_01 ;

drammar:hasMessage "Where is your father?"^^xsd:string rdfs:comment "Hamlet asks to Ophelia 'Where is your Father?' " $\wedge \wedge x$ sd:string .

\#\#\# http://www.pur1.org/drammar\#TL_Unit11

drammar:TL Unit11 rdf:type owl:NamedIndividual, drammar:Timeline ;

drammar:hasTimelineEffect drammar:CSS_Eff_TL_Unit11 ;

\#\#\# http://www.purl.org/drammar\#CSS_Eff_TL_Unit11

drammar:CSS_Eff_TL_Unit11 raf:type owl:NamedIndividual , drammar:Consistentstateset.

\#\#\# http://www.purl.org/drammar\#Unit11_Lie

drammar:Unit11_Lie rdf:type owl:NamedIndividual , drammar:Unit ; rdfs:comment "O. lies about her father's position."^^xsd:string . 
\#\#\# http://www.pur1.org/drammar\#A_Lying

drammar:A_Lying raf:type owl:NamedIndividual, drammar:Action ; drammar:hasMessage "At home, my lord"^^xsd:string ; drammar:isDescribedBy drammar:PS_prevarication_01 ; rdfs:comment "Ophelia lies about Polonius' location"^^xsd:string .

\section{\#\#\# http://www.purl.org/drammar\#Hamlet_Honesty_Atstake}

drammar:Hamlet_Honesty_AtStake rdf:type owl:NamedIndividual , drammar:Value ;

drammar:isDescribedBy drammar:VS_H_Honesty ;

drammar:atstake "true"^^xsd:boolean.

\section{\#\#\# http://www.purl.org/drammar\#Hamlet}

drammar:Hamlet raf:type owl:NamedIndividual , drammar:Agent ; drammar: hasExtRef drammar:ExtRef_prince ; drammar:hasGoal drammar:Goal_Hamlet_AskingRethorically ; drammar:intends drammar:P_H_AskR drammar:P_H_End_P_H_Learning_Honesty ; drammar:hasvalueEngaged drammar:Hamlet_Honesty_Atstake ; rdfs:comment "Hamlet Prince of Denmark"^^xsd:string.

\section{\#\#\# http://www.purl.org/drammar\#Ophelia}

drammar:Ophelia rdf:type owl:NamedIndividual drammar: Agent ; drammar:hasExtRef drammar:ExtRef_girlfriend ; drammar:hasGoal drammar:Goal_ophelia_Lying ; drammar:intends drammar:P_O_Lie, drammar:P_O_Saving_Polonius_Authority ; rdfs:comment "Ophelia, Daughter of Polonius"^^xsd:string .

\section{References}

Abdallah, S.A. \& Ferris, B. (2010). The ordered list ontology 0.72. Available at: http://smiy.sourceforge.net/olo/spec/ orderedlistontology.html.

Albert, G., Pizzo, A., Lombardo, V., Damiano, R. \& Terzulli, C. (2016). Bringing authoritative models to computational drama (encoding Knebel's action analysis). In Interactive Storytelling. 9th International Conference on Interactive Digital Storytelling, ICIDS 2016 (Vol. 10045, pp. 285-297). Cham: Springer International Publishing. doi:10.1007/978-3-319-48279$8 \_25$.

Archer, W. (1912). Play-Making: A Manual of Craftsmanship. Chapman \& Hall.

Aristotle (2013). Poetics. Oxford University Press.

Baker, C.F., Fillmore, C.J. \& Lowe, J.B. (1998). The Berkeley framenet project. In Proceedings of the 36th Annual Meeting of the Association for Computational Linguistics and 17th International Conference on Computational Linguistics (Vol. 1, pp. 86-90). Association for Computational Linguistics.

Battaglino, C., Damiano, R. \& Lesmo, L. (2013). Emotional range in value-sensitive deliberation. In Proceedings of the 2013 International Conference on Autonomous Agents and Multi-Agent Systems (pp. 769-776). International Foundation for Autonomous Agents and Multiagent Systems.

Bazin, A. \& Gray, H. (1967). What Is Cinéma?: Essays Selected and Translated by Hugh Gray. University of California Press. 
Berners-Lee, T., Hendler, J., Lassila, O., et al. (2001). The semantic web. Scientific American, 284(5), 28-37. doi:10.1038/ scientificamerican0501-34.

Bizer, C., Lehmann, J., Kobilarov, G., Auer, S., Becker, C., Cyganiak, R. \& Hellmann, S. (2009). DBpedia - a crystallization point for the web of data. Web Semantics: Science, Services and Agents on the World Wide Web, 7(3), 154-165.

Bloom, H. (1998). Shakespeare: The Invention of the Human. New York: Riverhead Books.

Bratman, M.E. (1987). Intentions, Plans, and Practical Reason. Harvard (Massachusetts): Harvard University Press.

Bratman, M.E., Israel, D.J. \& Pollack, M.E. (1988). Plans and resource-bounded practical reasoning. Computational Intelligence, 4, 349-355. doi:10.1111/j.1467-8640.1988.tb00284.x.

Brooks, C. \& Heilmann, R.B. (1946). Understanding Drama. G.G. Harrap.

Brunetière, F. (1914). The Law of the Drama. Dramatic Museum of Columbia University.

Carnicke, S.M. (2010). The Knebel technique: Active analysis in practice. In Actor Training (pp. 99-116).

Cataldi, M., Damiano, R., Lombardo, V. \& Pizzo, A. (2013). Lexical mediation for ontology-based annotation of multimedia. In New Trends of Research in Ontologies and Lexical Resources (pp. 113-134). Berlin, Heidelberg: Springer. doi:10.1007/ 978-3-642-31782-8_7.

Cavazza, M. \& Pizzi, D. (2006). Narratology for interactive storytelling: A critical introduction. In Technologies for Interactive Digital Storytelling and Entertainment (pp. 72-83). Springer. doi:10.1007/11944577_7.

Ciotti, F. (2016). Toward a formal ontology for narrative. MATLIT: Materialities of Literature, 4(1), 29-44. doi:10.14195/21828830_4-1_2.

Cuddon, J.A. (2012). A Dictionary of Literary Terms and Literary Theory. John Wiley \& Sons.

Currie, G. (2009). Narrative and the psychology of character. Journal of Aesthetics and Art Criticism, 67(1), 61-71. doi:10. 1111/j.1540-6245.2008.01335.x.

Damiano, R. \& Pizzo, A. (2008). Emotions in drama characters and virtual agents. In AAAI Spring Symposium on Emotion, Personality, and Social Behavior (pp. 30-37).

Dennett, D. (1987). The Intentional Stance. Cambridge (MA): MIT Press.

Diderot, D. (1966). Selected Writings. Classics in the History of Thought. Macmillan.

Doerr, M. (2003). The CIDOC conceptual reference module: An ontological approach to semantic interoperability of metadata. AI Magazine, 24(3), 75.

Doerr, M. \& LeBoeuf, P. (2007). Modelling intellectual processes: The FRBR - CRM harmonization. In C. Thanos, F. Borri and L. Candela (Eds.), Digital Libraries: Research and Development. Lecture Notes in Computer Science (Vol. 4877, pp. 114123). Berlin, Heidelberg: Springer. doi:10.1007/978-3-540-77088-6_11.

Doty, C. (2013). The difficulty of an ontology of live performance. InterActions: UCLA Journal of Education and Information Studies, 9(1).

Eger, M., Barot, C. \& Young, R.M. (2015). Impulse: A formal characterization of story. In 6th Workshop on Computational Models of Narrative (p. 45).

Egri, L. (1946). The Art of Dramatic Writing. New York: Simon and Schuster.

Elam, K. (1980). The Semiotics of Theatre and Drama. London and New York: Methuen.

Elson, D.K. (2012). DramaBank: Annotating agency in narrative discourse. In Proceedings of the Eighth International Conference on Language Resources and Evaluation (LREC 2012), Istanbul, Turkey.

Esslin, M. (1988). The Field of Drama. London: Methuen. (First published in 1987 ed.).

Fairclough, C.R. (2004). Story games and the OPIATE system: Using case-based planning for structuring plots with an expert story director agent and enacting them in a socially simulated game world. Doctoral Thesis, University of Dublin - Trinity College.

Ferrario, R. \& Oltramari, A. (2004). Towards a computational ontology of mind. In A.C. Varzi and L. Vieu (Eds.), Proceedings of the International Conference (FOIS 2004), Torino, Italy (pp. 287-297).

Field, S. (2003). The Definitive Guide to Screen Writing. London: Ebury Press.

Fikes, R.E. \& Nilsson, N.J. (1971). STRIPS: A new approach to the application of theorem proving to problem solving. Artificial Intelligence, 2, 189-208. doi:10.1016/0004-3702(71)90010-5.

Fillmore, C.J. (1985). Frames and the semantics of understanding. Quaderni di semantica, 6.

François, A.R.J., Nevatia, R., Hobbs, J. \& Bolles, R.C. (2005). VERL: An ontology framework for representing and annotating video events. IEEE MultiMedia, 5, 76-86. doi:10.1109/MMUL.2005.87.

Freytag, G. (1863). Technique of the Drama, an Exposition of Dramatic Composition and Art. Chicago: S.C. Griggs and Company. (Reprint 1985 ed.).

Gangemi, A. (2005). Ontology design patterns for semantic web content. In The Semantic Web - ISWC 2005: 4th International Semantic Web Conference, ISWC 2005, Proceedings, Galway, Ireland, November 6-10, 2005 (Vol. 3729, p. 262). Springer.

Gangemi, A. \& Presutti, V. (2009). Ontology design patterns. In Handbook on Ontologies (pp. 221-243). doi:10.1007/978-3540-92673-3_10.

George Lukács, L.B. (1965). The sociology of modern drama. The Tulane Drama Review, 9(4), 146-170. http://www.jstor.org/ stable/1125039. doi:10.2307/1125039. 
Gervás, P. (2013). Propp's morphology of the folk tale as a grammar for generation. In Proc. of the Int. Workshop on Computational Models of Narrative (CMN13) (pp. 106-122).

Gervás, P., Díaz-Agudo, B., Peinado, F. \& Hervás, R. (2005). Story plot generation based on CBR. Knowledge-Based Systems, 18(4), 235-242. doi:10.1016/j.knosys.2004.10.011.

Greimas, A.J. (1977). Elements of a narrative grammar. Diacritics, 23-40. doi:10.2307/464872.

Guarino, N. \& Welty, C.A. (2009). An overview of OntoClean. In Handbook on Ontologies (pp. 201-220). Springer. doi:10. 1007/978-3-540-92673-3_9.

Hartmann, K., Hartmann, S. \& Feustel, M. (2005). Motif definition and classification to structure non-linear plots and to control the narrative flow in interactive dramas. In Virtual Storytelling, Using Virtual Reality Technologies for Storytelling, Third International Conference, ICVS (pp. 158-167). doi:10.1007/11590361_18.

Hegel, G.W.F. (1885). Hegel's Aesthetics. S.C. Griggs and Company.

Hoffart, J., Suchanek, F.M., Berberich, K. \& Weikum, G. (2013). YAGO2: A spatially and temporally enhanced knowledge base from Wikipedia. Artificial Intelligence, 194, 28-61. doi:10.1016/j.artint.2012.06.001.

Horace (1989). Epistles, Book II; and Epistle to the Pisones (Ars Poetica). N. Rudd (Ed.). Cambridge: Cambridge University Press.

Jewell, M.O., Lawrence, K.F., Tuffield, M.M., Prugel-Bennett, A., Millard, D.E., Nixon, M.S., Schraefel, M.C. \& Shadbolt, N.R. (2005b). OntoMedia: An ontology for the representation of heterogeneous media. In Multimedia Information Retrieval Workshop (MMIR 2005) SIGIR. ACM SIGIR.

Jewell, M.O., Lawrence, K.F., Tuffield, M.M., Prugel-Bennett, A., Millard, D.E., Nixon, M.S., Shadbolt, N.R., et al. (2005a). OntoMedia: An ontology for the representation of heterogeneous media. In Proceeding of SIGIR Workshop on Mutlimedia Information Retrieval. ACM SIGIR.

Lavandier, Y. (1994). La Dramaturgie. Cergy: Le clown et l'enfant.

Lawrence, K.F. (2011). Crowdsourcing linked data from Shakespeare to Dr who. In Web Science International Conference.

Lessing, G.E. (1962). Hamburg Dramaturgy. Dover Publications.

Lombardo, V., Battaglino, C., Pizzo, A., Damiano, R. \& Lieto, A. (2015b). Coupling conceptual modeling and rules for the annotation of dramatic media. Semantic Web, 6(5), 503-534. doi:10.3233/SW-140156.

Lombardo, V., Damiano, R., Battaglino, C. \& Pizzo, A. (2015a). Automatic annotation of characters' emotions in stories. In International Conference on Interactive Digital Storytelling (pp. 117-129). Springer. doi:10.1007/978-3-319-27036-4_11.

Lombardo, V., Damiano, R. \& Pizzo, A. (2018). Drammar: A comprehensive ontological resource on drama. In International Semantic Web Conference (pp. 103-118). Springer.

Lombardo, V., Damiano, R., Pizzo, A. \& Terzulli, C. (2017b). The intangible nature of drama documents: An FRBR view. In Proceedings of the 2017 ACM Symposium on Document Engineering (pp. 173-182). ACM.

Lombardo, V., Damiano, R., Pizzo, A., Terzulli, C., Ceccaldi, E., Albert, G. \& Pulizzotto, D. (2017a). Annotation of metadata for dramatic texts: The POP-ODE initiative. In Proceedings of the 1st Workshop on Natural Language for Artificial Intelligence Co-Located with 16th International Conference of the Italian Association for Artificial Intelligence (Vol. 1983, pp. 30-42). CEUR-WS.

Lombardo, V. \& Pizzo, A. (2016). Multimedia tool suite for the visualization of drama heritage metadata. Multimedia Tools and Applications, 75(7), 3901-3932. doi:10.1007/s11042-014-2066-3.

Lombardo, V., Pizzo, A. \& Damiano, R. (2016). Safeguarding and accessing drama as intangible cultural heritage. Journal on Computing and Cultural Heritage (JOCCH), 9(1), 5.

Masolo, C., Borgo, S., Gangemi, A., Guarino, N., Oltramari, A., Oltramari, R., Schneider, L., Istc-cnr, L.P. \& Horrocks, I. (2002). WonderWeb deliverable D17. The WonderWeb Library of Foundational Ontologies and the DOLCE ontology.

McCarthy, A. (1986). Mental situation calculus. In TARK: Theoretical Aspects of Reasoning About Knowledge.

McKee, R. (1997). Story. New York: Harper Collins.

Miller, G.A. (1995). WordNet: A lexical database for English. Communications of the ACM, 38(11), 39-41. doi:10.1145/ 219717.219748.

Minsky, M. (1977). Frame-system theory. In Thinking: Readings in Cognitive Science (pp. 355-376).

Motik, B., Grau, B.C., Horrocks, I., Wu, Z., Fokoue, A. \& Lutz, C. (2009a). Owl 2 web ontology language: Profiles. W3C Recommendation, 27, 61.

Motik, B., Patel-Schneider, P.F., Parsia, B., Bock, C., Fokoue, A., Haase, P., Hoekstra, R., Horrocks, I., Ruttenberg, A., Sattler, U., et al. (2009b). Owl 2 web ontology language: Structural specification and functional-style syntax. W3C Recommendation, 27(65), 159.

Mulholland, P. \& Collins, T. (2002). Using digital narratives to support the collaborative learning and exploration of cultural heritage. In Database and Expert Systems Applications, 2002. Proceedings. 13th International Workshop on (pp. 527-531). IEEE.

Naphade, M., Smith, J.R., Tesic, J., Chang, S.-F., Hsu, W., Kennedy, A.L., Hauptmann, A. \& Curtis, J. (2006). Large-scale concept ontology for multimedia. IEEE Multimedia, 13(3), 86-91. doi:10.1109/MMUL.2006.63. 
Nau, D., Cao, Y., Lotem, A. \& Munoz-Avila, H. (1999). SHOP: Simple hierarchical ordered planner. In Proceedings of the 16th International Joint Conference on Artificial Intelligence (Vol. 2, pp. 968-973). Morgan Kaufmann Publishers Inc.

Newman, I. (2009). Virtual people: Fictional characters through the frames of reality. Journal of Aesthetics and Art Criticism, 67(1), 73-82. doi:10.1111/j.1540-6245.2008.01336.x.

Noy, N.F., Mortensen, J., Alexander, P.R. \& Musen, M.A. (2013). Mechanical Turk as an ontology engineer? Using microtasks as a component of an ontology engineering workflow. In Web Science.

Olson, E. (1961). Tragedy and the Theory of Drama. Wayne Books. Wayne State University Press.

O'Neill, E.T. (2011). Frbr: Functional requirements for bibliographic records. Library Resources \& Technical Services, 46(4), 150-159. doi:10.5860/1rts.46n4.150.

Ortony, A., Clore, G. \& Collins, A. (1988). The Cognitive Structure of Emotions. Cambridge University Press.

Pease, A., Niles, I. \& Li, J. (2002). The suggested upper merged ontology: A large ontology for the semantic web and its applications. In Working Notes of the AAAI - 2002 Workshop on Ontologies and the Semantic Web (Vol. 28). Canada: Edmonton.

Pfister, M. (1991). The Theory and Analysis of Drama. European Studies in English Literature. Cambridge University Press.

Pianta, E., Bentivogli, L. \& Girardi, C. (2002). MultiWordNet: Developing an aligned multilingual database. In Proceedings of the First International Conference on Global WordNet. Available at: http://multiwordnet.fbk.eu/paper/MWN-Indiapublished.pdf.

Polti, G. (1895). Les Trente-Six Situations Dramatiques. Paris: Mercure de France.

Propp, V. (1968). Morphology of the Folktale. University of Texas Press.

Rahimtoroghi, E., Corcoran, T., Swanson, R., Walker, M.A., Sagae, K. \& Gordon, A. (2014). Minimal narrative annotation schemes and their applications. In Seventh Intelligent Narrative Technologies Workshop.

Rao, A. \& Georgeff, M. (1991). Modeling rational agents within a BDI architecture. In Proceedings of KR'91 (pp. 473-484). Morgan Kaufmann.

Ryngaert, J.P. (2008). Introduction à L'analyse du Théâtre. Collection Cursus. Série Littérature. Armand Colin.

Sardina, S., de Silva, L. \& Padgham, L. (2006). Hierarchical planning in BDI agent programming languages: A formal approach. In Proceedings of the Fifth International Joint Conference on Autonomous Agents and Multi-Agent Systems (pp. 1001-1008). ACM.

Schank, R.C. \& Abelson, R.P. (1975). Scripts, Plans, and Knowledge. New Haven, CT: Yale University.

Seger, L. (1990). Creating Unforgettable Characters. New York: Henry Holt and Company.

Shoham, Y. (1987). Reasoning About Change. Cambridge, MA: MIT Press.

Simperl, E. \& Luczak-Rösch, M. (2014). Collaborative ontology engineering: A survey. The Knowledge Engineering Review, 29(01), 101-131. doi:10.1017/S0269888913000192.

Spencer, S. (2002). The Playwright's Guidebook: An Insightful Primer on the Art of Dramatic Writing. Faber \& Faber.

Styan, J.L. (1963). The Elements of Drama. Cambridge: University Press.

Suarez-Figueroa, M.C., Gomez-Perez, A. \& Fernandez-Lopez, M. (2012). The NeOn methodology for ontology engineering. In Ontology Engineering in a Networked World (pp. 9-34). Springer.

Sure, Y., Staab, S. \& Studer, R. (2004). On-to-knowledge methodology (OTKM). In Handbook on Ontologies (pp. 117-132). Springer. doi:10.1007/978-3-540-24750-0_6.

Sweet, J. (1993). The Dramatist's Toolkit: The Craft of the Working Playwright. Portsmouth, N.H.: Heinemann.

Szilas, N. (2016). Modeling and representing dramatic situations as paradoxical structures. Digital Scholarship in the Humanities, 071.

Szilas, N., Estupiñán, S. \& Richle, U. (2016). Qualifying and quantifying interestingness in dramatic situations. In Interactive Storytelling: 9th International Conference on Interactive Digital Storytelling, ICIDS 2016, Proceedings Los Angeles, CA, USA, November 15-18, 2016 (Vol. 9, pp. 336-347). Springer. doi:10.1007/978-3-319-48279-8_30.

Szondi, P. (1983). Theory of the moderna drama parts I-II. boundary 2, 11(3), 191-230).

Tomaszewski, Z. \& Binsted, K. (2007). The limitations of a Propp-based approach to interactive drama. In Intelligent Narrative Technologies: Papers from the AAAI Fall Symposium (Vol. FS-07-05, pp. 167-173). AAAI Press.

van Riemsdijk, M.B., Dastani, M. \& Meyer, J.-J.C. (2009). Goals in conflict: Semantic foundations of goals in agent programming. Autonomous Agents and Multi-Agent Systems, 18(3), 471. doi:10.1007/s10458-008-9067-4.

Varela, M.E. (2016). Interoperable performance research: Promises and perils of the semantic web. TDR/The Drama Review, 60(3), 136-147. doi:10.1162/DRAM_a_00574.

Wolff, A., Mulholland, P. \& Collins, T. (2012). Storyspace: A story-driven approach for creating museum narratives. In Proceedings of the 23rd ACM Conference on Hypertext and Social Media (pp. 89-98). ACM.

Zarri, G.P. (1997). NKRL, a knowledge representation tool for encoding the 'meaning' of complex narrative texts. Natural Language Engineering, 3(2), 231-253. doi:10.1017/S1351324997001794.

Zarri, G.P. (2014). Conceptual and content-based annotation of (multimedia) documents. Multimedia Tools and Applications, 72(3), 2359-2391. doi:10.1007/s11042-013-1463-3. 